Modeling, Identification and Control, Vol. 30, No. 2, 2009, pp. 39-56, ISSN 1890-1328

\title{
Kinematic and Elastostatic Design Optimisation of the 3-DOF Gantry-Tau Parallel Kinematic Manipulator
}

\author{
Ilya Tyapin ${ }^{1}$ Geir Hovland ${ }^{2}$
}

\author{
${ }^{1}$ School of Information Technology and Electrical Engineering, The University of Queensland, Brisbane, Queens- \\ land, 4001, Australia. E-mail: ilya@itee.uq.edu.au \\ ${ }^{2}$ Department of Engineering, Mechatronics Group, University of Agder, N-4898 Grimstad, Norway. E-mail: \\ geir.hovland@uia.no
}

\begin{abstract}
One of the main advantages of the Gantry-Tau machine is a large accessible workspace/footprint ratio compared to many other parallel machines. The Gantry-Tau improves this ration further by allowing a change of assembly mode without internal link collisions or collisions between the links and end-effector. The reconfigurable Gantry-Tau kinematic design obtained by multi-objective optimisation according to this paper gives the following features: 3-D workspace/footprint ratio is more than 3.19, lowest Cartesian stiffness in the workspace is $5 \mathrm{~N} / \mu \mathrm{m}$ and no link collisions detected. The optimisation parameters are the support frame lengths, the actuator positions and the robot's arm lengths. The results comparison between the evolutionary complex search algorithm and gradient-based method used for the Gantry-Tau design in the past is also presented in this paper. The detailed statics model analysis of the Gantry-Tau based on a functionally dependency is presented in this paper for the first time. Both the statics model and complex search algorithm may be applied for other 3-DOF Hexapods without major changes. The existing lab prototype of the Gantry-Tau was assembled and completed at the University of Agder, Norway.
\end{abstract}

Keywords: parallel manipulator, statics, design optimisation.

\section{Introduction}

A generalised parallel kinematic manipulator (PKM) is a closed-loop kinematic chain mechanism where the end-effector is linked to the base by several independent kinematic chains, (Merlet, 2000). It may consist of redundant mechanisms with more actuators than the number of controlled degrees of freedom of the endeffector. The study of PKMs has been an active research field in robotics and mechanical design for a long time and different parallel mechanism with specified number and type of DOF have been proposed. In the late 1980s a new field of applications and research was developed by (Clavel, 1988), who put the focus on lower mobility parallel mechanism. The Delta robot presented by Clavel was a base for a large range of machines dedicated to high-speed applications but because of small workspace in relation to footprint and limited number of degrees of freedom the robot can not be used as a general purpose manipulator. Pierrot proposed a 6-DOF fully-parallel robot HEXA. The HEXA robot, (Pierrot et al., 1992) and (Uchiyama et al., 1990), is an extension of the Delta mechanism having 6 DOFs but because the workspace/footprint ratio and tilting angles are small this robot has also a limited capability compared to serial manipulators.

The Tau family of parallel kinematic manipulators was invented by ABB Robotics, see (Brogårdh, 2000). 


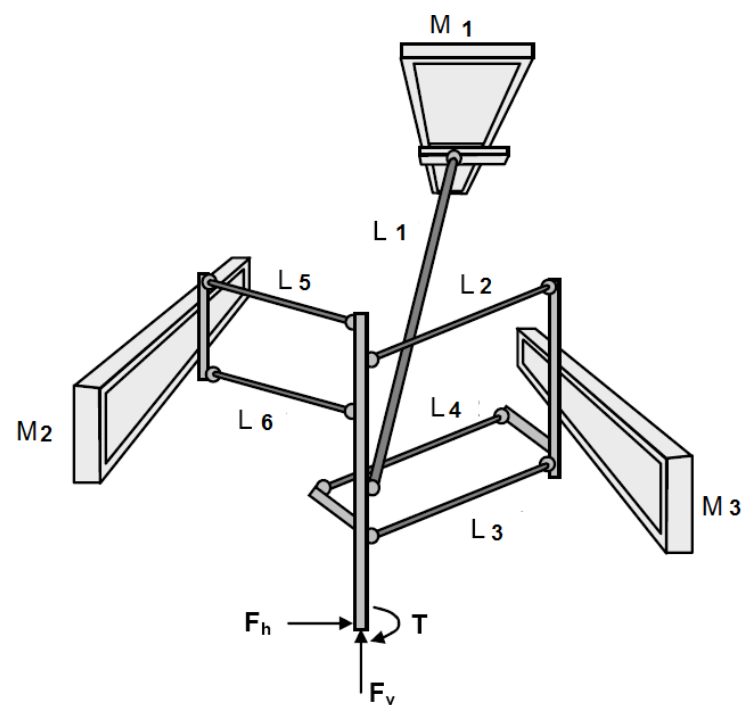

Figure 1: A variant of 3-2-1 link structure mounted on three quide ways.

The Gantry-Tau PKM makes use of the non-symmetric Tau structure that is based on clustering the links mounted on the actuated arms in the configuration 3-21. The different possibilities of Tau structures are presented in (Brogårdh and $\mathrm{Gu}, 2002$ ). The link structure 3-2-1 is shown in Fig. 1, where the six links support only axial forces. Tool forces (represented by $F_{h}$ ) in the figure, generate forces and torques on the manipulated platform (in the figure a shaft), which are fully supported by axial forces in the six links. The link structure 3-2-1 gives the best opportunities to adapt the PKM to the specific application requirements. The high stiffness is obtained by the redundant replacement of the linear actuator $M_{1}$ and position of the link $L_{1}$; the distance between links can be increased without reducing the workspace, it makes a possibility of reducing the link forces, especially with respect to the vertical forces on the TCP. The intended applications of the Gantry-Tau are high speed machining operations requiring a workspace equal to or larger than a typical serial-type robot's, but with higher stiffness. However, the robot can also be designed for very fast material handling and assembly or for high precision processes such as laser cutting, water jet cutting and measurement.

\subsection{Workspace}

In order to calculate the workspace one can employ discretisation methods, geometrical methods or numerical methods. For the discretisation method a grid of nodes with positions and orientations is defined. Then the kinematics is calculated for each node and it is straightforward to verify whether the kinematics can be solved and to check if joint limits are reached or link interference occurs. The discretisation algorithm is simple to implement but has some serious drawbacks. It is expensive in computation time and results are limited to the nodes of the grid. One example of this approach is (Dashy et al., 2002). The most common numerical methods are based upon the Newton-Raphson approach (Whitney, 1969) and its variants. Using geometrical methods the workspace can be calculated as an intersection of simple geometrical objects, (Merlet, 2000), for example spheres. The geometric approach to define the Gantry-Tau maximum workspace is presented in this paper.

\subsection{Link Collisions}

The distance between two geometric objects (lines, segments of lines, rays, surfaces etc.) is defined as the minimum distance between two points on these objects. Link collisions occur when the distance between two points on the links is less than the sum of radii of these links. The links of the manipulator are assumed to be cylindrical elements.

The link collisions detection methods were presented in (Eberly, 2001), (Merlet and Daney, 2006) and (Teller, 2008), but all use vector cross-products to define the closest distance between two line segments in $3 \mathrm{D}$ plane and no functional analysis of the distance function in 2D plane was attempted in either (Eberly, 2001) and (Teller, 2008). The Gantry-Tau link collisions detection presented in (Tyapin, 2009) is based on the conditional equations (boundaries) search method and included into the design optimisation scheme presented in this paper. The functional dependency analysis is applied to the condition equations.

\subsection{Stiffness}

The stiffness is the most important performance specification of parallel kinematic machines. Dimentberg (Dimentberg, 1965) was among the first to use screw theory to define the stiffness matrix of spring systems in unloaded equilibrium. A stiffness matrix relates external forces and torques to the linear and angular displacements of the joints in the Cartesian space. Since then, the Cartesian stiffness was studied and there are a lot of calculation methods and algorithms available such as Finite Element Analysis (Pashkevich et al., 2005), virtual joint method (Majou et al., 2004). The stiffness maps of the workspace through Jacobian Matrix was established in (Gosselin, 1999), (Bi et al., 2007). The stiffness in $X$-, $Y$ - and $Z$-directions were computed in (El-Khasawneh and Ferreira, 1999), where the minimum and maximum stiffness and directions in 
a given configuration were found through the eigenvectors analysis. An approach to get the stiffness model of a tripod-based PKM was presented in (Huang and Mei, 2001), where the machine structure is decomposed into two substructures: machine frame and parallel mechanism.

Two other interesting works considered in our study are (Li et al., 2002) and (Li and Kao, 2004). The authors proposed the conservative congruence transformation (CCT), which represents the stiffness control between the linear Cartesian space at the end-effector and joint space of a robotic manipulator. In addition, the CCT for the stiffness mapping between the cylindrical space and joint space was presented in ( $\mathrm{Li}$ et al., 2002) and Jacobian matrix was used to define the Cartesian stiffness. In (Li and Kao, 2004) CCT was used to define a kinematic solution for redundant manipulators. Note that in (Li and Kao, 2004) a new stiffness matrix $\mathbf{K}_{\mathbf{g}}$ is defined, which represents the changes in geometry through the differential Jacobian matrix, and externally applied forces. In Section 4 of this paper, the $\mathbf{K}_{\mathbf{g}}$ matrix is not taken into account, since the joint stiffness $\mathbf{K}_{\theta}$ dominates for small external forces.

The closest methods to this paper are presented in (Pashkevich et al., 2007), (Company et al., 2005) and (Liu et al., 2007). The analytical stiffness modelling is presented in (Company et al., 2005), where the method is based on classical mechanical tools and equations. The application of this method to lower mobility parallel mechanisms is more difficult than the one for Hexapods. For the method in (Company et al., 2005) forces and torques are applied on the endpoint. Another method is presented in (Pashkevich et al., 2007), where the Jacobian matrices and numerical calculations were used to define the stiffness. In addition, the design optimisation is presented in (Pashkevich et al., 2007). The stiffness functional dependency analysis is not presented in (Pashkevich et al., 2007), (Company et al., 2005) and (Liu et al., 2007). The randomised optimal design of PKMs based on control random search technique is presented in Lou et al. (2008), where the effective regular workspace is introduced. The main drawback of the method in Lou et al. (2008) is the use of stiffness as the dexterity constraint but not the objective and one objective function based on workspace is used in Lou et al. (2008). The drawback of the optimisation algorithm is a lack of ability to reach the global optimum if the user is not experienced in the design optimisation and can not setup a range of the parameter's limits. However, the method is good for the Delta-type PKMs.

Three different approaches to define the Cartesian stiffness of the triangular version of the Gantry-Tau were used before in (Brogårdh et al., 2005), (Williams et al., 2006) and (Hovland et al., 2007). The first method was based on the forward kinematics. The forward kinematics was required to be calculated in numerical form, but numerical solutions are computationally expensive. The second method was to use the Jacobian matrix derived from the inverse kinematics and matrix inversion. The second method is less computational expensive than the first. The third method was to use the static matrix and avoid matrix inversions to calculate the Cartesian stiffness in (Hovland et al., 2007). The method presented in this paper is based on the geometric algebra and functional dependency analysis to calculate the static matrix and is an extension of the work in (Hovland et al., 2007). The main benefit of the static analysis presented in this paper is the savings in computational effort. The stiffness analysis is developed for the Gantry-Tau but may be used for the other Hexapods with minor changes.

\subsection{Multi-Objective Optimisation}

The design optimisation may have many objectives which cannot be found numerically and it is a reason why most proposed optimal design procedures are focused on the optimisation of the main characteristic of the manipulator, for example, workspace, conditioning and stiffness indices, vibration analysis and manipulability criteria. In Stamper et al. (1997) it is stated that a parallel manipulator with maximum possible workspace may have undesirable characteristics such as low stiffness or resonance frequencies, which means that a multi-objective optimisation is needed. The multi-objective design optimisation problem is expressed as follows.

$$
\begin{aligned}
& \min \left[f_{1}(\mathbf{p}), \ldots, \quad f_{k}(\mathbf{p}), f_{k+1}(\mathbf{p})\right] \\
& \text { subject to } \\
& g_{i}(\mathbf{p}) \leq 0 \quad(1 \leq i \leq r) \\
& h_{j}(\mathbf{p})=0 \quad(1 \leq j \leq s) \\
& p_{n}^{L} \leq p_{n} \leq p_{n}^{U} \quad(1 \leq n \leq m)
\end{aligned}
$$

where $k$ is the number of objective functions $f_{k}(\mathbf{p}), \mathbf{p}$ is a vector of $m$ optimisation parameters, $g_{i}(\mathbf{p})$ and $h_{j}(\mathbf{p})$ are each of the $r$ inequality and $s$ equality problem constraints, $p^{L}$ and $p^{U}$ are lower and upper limits of the optimisation parameters. The constraints are considered as a new objective constraints handling function $f_{k+1}(\mathbf{p})$. Different constraint handling techniques are presented in Coello (2002).

The most common approach for the constrains handling is the use of penalty functions. When using a penalty function, the constraint evaluation is used to penalise an infeasible solution and feasible solutions 
are favored by the selection process. However, penalty functions have several drawbacks, for example, they require a careful tuning of the penalty factors that accurately estimates the level of penalisation.

The complex search method was used for the mechanical design optimisation in Hansen et al. (2004) and Hansen and Andersen (2001). In Hansen and Andersen (2001) a mechanical design optimisation of a hydraulically actuated manipulator is presented. The main objective is minimising the energy consumption with side constraints on stability, response time and load dependency. The initial population has 30 designs and optimum design is found in 250 iterations. In Hansen et al. (2004) a multi-objective design optimisation of a servo-robot for a pallets handling is presented. The objectives are the cost and speed. The accuracy of the tool point, an expected life of the planetary gears and the welded structure, vibrations and thermal conditions of the servo motors are the main side constraints. Discrete design variables originally handled by a mapping technique. The optimum design was found in 50 iterations with an initial population of 10 .

In multi-objective optimisation, objectives are not comparable with respect to their magnitude and value and may conflict, where some objectives can not be increased without a decreasing of others. The result of a multi-objective optimisation is a set of trade-off solutions which are considered to be suitable for all objectives. In this paper the use of the evolutionary complex search algorithm for the PKM's multi-objective design optimisation is presented. The optimisation scheme includes the kinematic, elastostatic properties of the machine and constraint handling based on the penalisation function.

In Section 2 the kinematic description of the 3DOF Gantry-Tau parallel kinematic machine is presented. In Section 3 the workspace and unreachable areas caused by the collisions between the manipulated platform and support frame are presented. In Section 4 a geometric description of the Cartesian stiffness is presented. The parabolic functional dependency method to calculate the Cartesian stiffness in the $Y$-direction is presented in Section 4.1. The method to define the Cartesian stiffness in the $Z$-direction is presented in Section 4.2. In Section 4.3 the method for the stiffness in the $X$-direction is presented. In Section 5 the 3-DOF Gantry-Tau optimisation problem description and complex search algorithm are presented. In Sections 7 and 6 the results, conclusions and the future research directions are presented.

\section{Kinematic Description of the 3-DOF Gantry-Tau Parallel Kinematic Manipulator}

The triangular-link version of the Gantry-Tau kinematic model is illustrated in Figs. 2, 5 and 8. The 3DOF Gantry-Tau can be manually reconfigured while avoiding singularities. As for the basic Gantry-Tau structure, each of the 3 parallel arms (lengths $L_{1}, L_{2}$ and $\left.L_{3}\right)$ is controlled by a linear actuator with actuation variables $q_{1}, q_{2}$ and $q_{3}$. The actuators in Fig. 2 are aligned in the direction of the global $X$-coordinate.

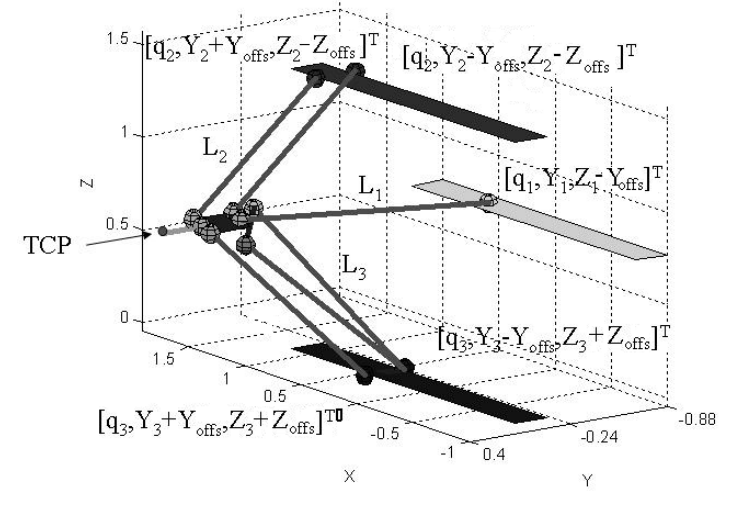

Figure 2: Triangular-link variant of the Gantry-Tau shown in the left-handed configuration for all link clusters.

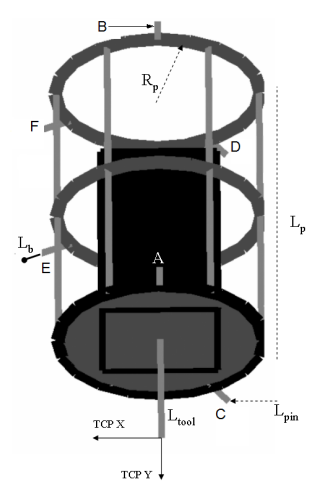

Figure 3: The manipulated platform of the Gantry-Tau robot.

Figs. 3 and 4 show the manipulated platform. The points $A, B, C, D, E$ and $F$ are the link connection points. The arm with one single link connects the actuator $q_{1}$ with platform point $F$. The arm with two links connects actuator $q_{2}$ with the platform points $A$ and $B$. The arm with three links connects actuator $q_{3}$ with the platform points $C, D$ and $E$. The triangular pair 
is connected to points $D$ and $E$. Fig. 7 shows a projection of the link system in Fig. 2 into the $X Z$-plane and Fig. 6 a projection into the $Y Z$-plane. Fig. 3 shows

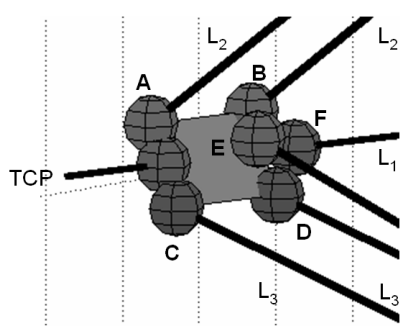

Figure 4: Kinematic platform parameters.

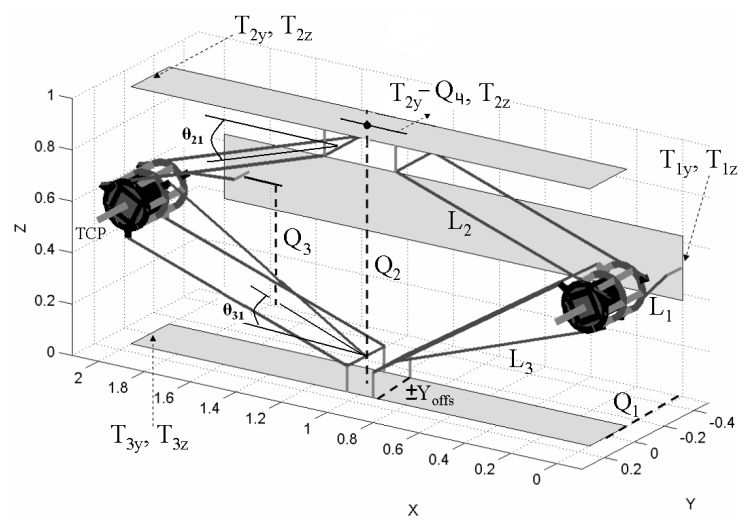

Figure 5: The 3-DOF reconfigurable Gantry-Tau robot.

the fixed kinematic parameters of the moving platform, which are not included in the design optimisation. $L_{p}$ is the platform length, $R_{p}$ is the platform radius, $L_{t o o l}$ is the tool length, $L_{\text {pin }}$ is the length from the platform circle of radius $R_{p}$ to the connection point for the universal joints and $L_{b}$ is the length from the connection point to the centre of the joint. According to Fig. 4, the single link with length $L_{1}$ is connected to the pin $F$ via a universal joint pin. The TCP of the robot is located at the tip of the milling tool. Each link has a passive $2 \mathrm{D}$ universal joint connected to the actuator and passive $3 \mathrm{D}$ joint connected to the platform.

Fig. 5 shows the PKM structure in both the leftand right-handed assembly modes. The Tau structure is characterised by a clustering of the links in groups of 1, 2 and 3, respectively, with fixed link lengths $L_{1}$, $L_{2}$ and $L_{3}$. Three linear actuators are used at the base to move the three arms independently in the global $X$ direction. The actuator track locations are fixed in the $Y$ - and $Z$-directions and the locations are denoted $T_{1 y}$, $T_{1 z}, T_{2 y}, T_{2 z}, T_{3 y}$ and $T_{3 z}$, respectively (see Figs. 2,5 and 6 ). The dimensioning of the PKM's support frame is given by variables $Q_{1}, Q_{2}, Q_{3}$ and $Q_{4}$ as illustrated in Fig. 5, where $Q_{1}$ is the depth and $Q_{2}$ is the height,
$Q_{3}$ is the $Z$-coordinate of the actuator $T_{1}$ and $Q_{4}$ is the $Y$-coordinate of the actuator $T_{2}$.

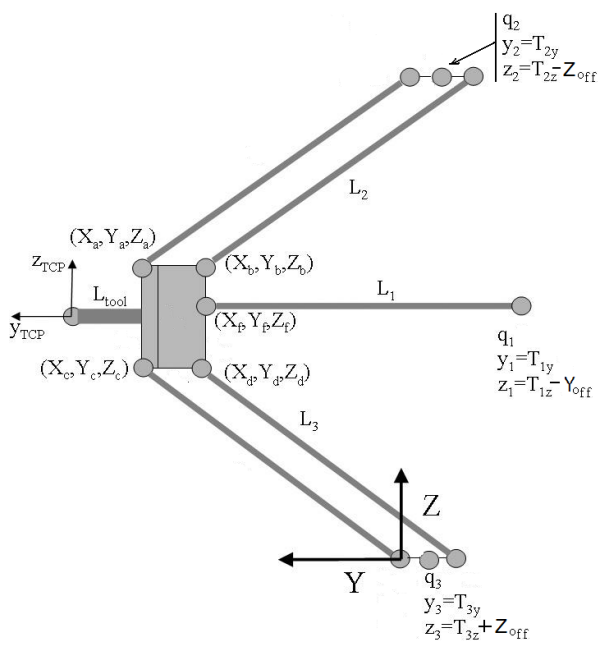

Figure 6: Kinematic parameters in YZ-plane.

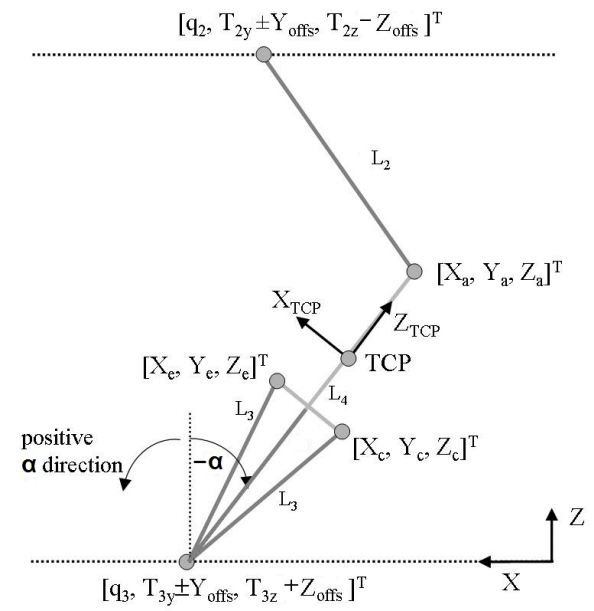

Figure 7: Kinematic parameters in XZ-plane.

The normalised vectors pointed from the actuator positions to the points $A, B, C, D, E, F$ on the platform 


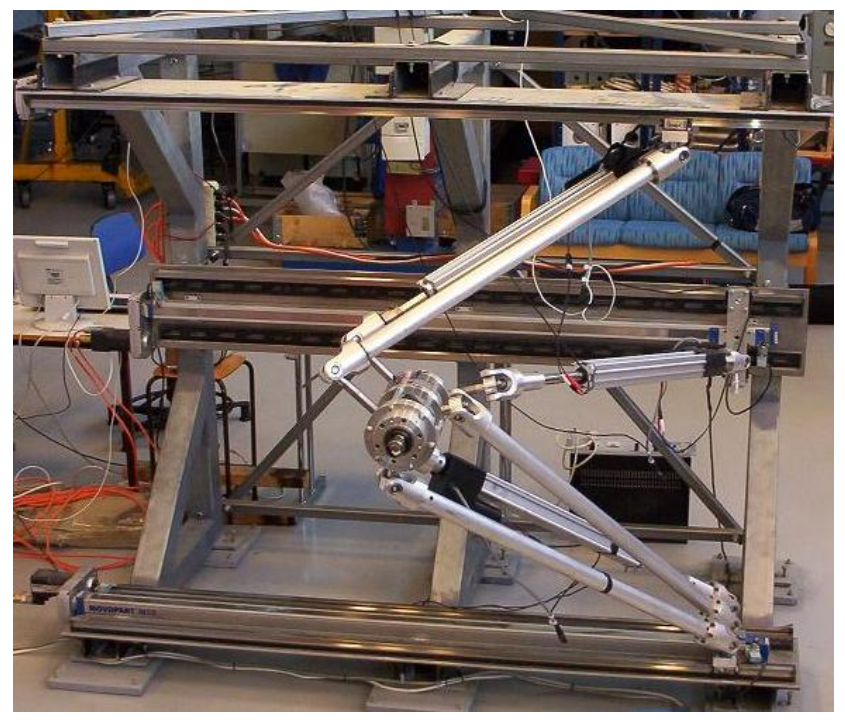

Figure 8: A prototype of the Gantry-Tau with a triangular-mounted link pair built at the University of Agder.

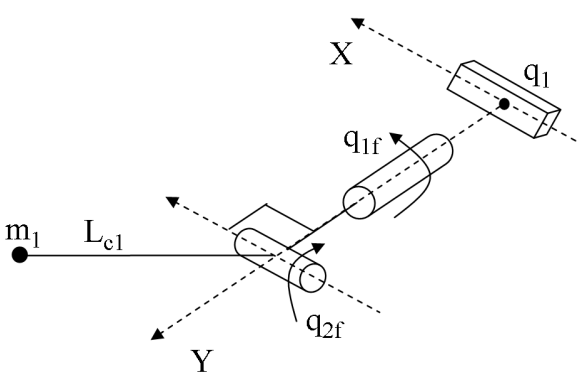

Figure 9: Definitions of the variables at the actuator side for the single link.

are given below.

$$
\begin{gathered}
\mathbf{A}=\left[\begin{array}{lll}
A_{x} & A_{y} & A_{z}
\end{array}\right]^{T} \quad \mathbf{B}=\left[\begin{array}{lll}
B_{x} & B_{y} & B_{z}
\end{array}\right]^{T} \\
\mathbf{C}=\left[\begin{array}{lll}
C_{x} & C_{y} & C_{z}
\end{array}\right]^{T} \quad \mathbf{D}=\left[\begin{array}{lll}
D_{x} & D_{y} & D_{z}
\end{array}\right]^{T} \\
\mathbf{E}=\left[\begin{array}{lll}
E_{x} & E_{y} & E_{z}
\end{array}\right]^{T} \quad \mathbf{F}=\left[\begin{array}{lll}
F_{x} & F_{y} & F_{z}
\end{array}\right]^{T}
\end{gathered}
$$

$\mathbf{A}=\left[\left(a_{x} C+a_{z} S+d X_{1}\right)\left(a_{y}+d Y_{1}\right)\left(a_{z} C-a_{x} S+d Z_{1}\right)\right]^{T}$

$\mathbf{B}=\left[\left(b_{x} C+b_{z} S+d X_{2}\right)\left(b_{y}+d Y_{2}\right)\left(b_{z} C-b_{x} S+d Z_{2}\right)\right]^{T}$

$\mathbf{C}=\left[\left(c_{x} C+c_{z} S+d X_{3}\right)\left(c_{y}+d Y_{3}\right)\left(c_{z} C-c_{x} S+d Z_{3}\right)\right]^{T}$

$\mathbf{D}=\left[\left(d_{x} C+d_{z} S+d X_{4}\right)\left(d_{y}+d Y_{4}\right)\left(d_{z} C-d_{x} S+d Z_{4}\right)\right]^{T}$

$\mathbf{E}=\left[\left(e_{x} C+e_{z} S+d X_{5}\right)\left(e_{y}+d Y_{5}\right)\left(e_{z} C-e_{x} S+d Z_{5}\right)\right]^{T}$

$\mathbf{F}=\left[\left(f_{x} C+f_{z} S+d X_{6}\right)\left(f_{y}+d Y_{6}\right)\left(f_{z} C-f_{x} S+d Z_{6}\right)\right]^{T}$

where $C=\cos \alpha, S=\sin \alpha, \alpha$ is the platform orientation angle and shown in Fig. $7, d X_{i}=X-T_{i x}$, $d Y_{i}=Y-T_{i y}, d Z_{i}=Z-T_{i z}$, where $T_{i x}, T_{i y}, T_{i z}$ are the coordinates of actuator $i$ for the given TCP posi-

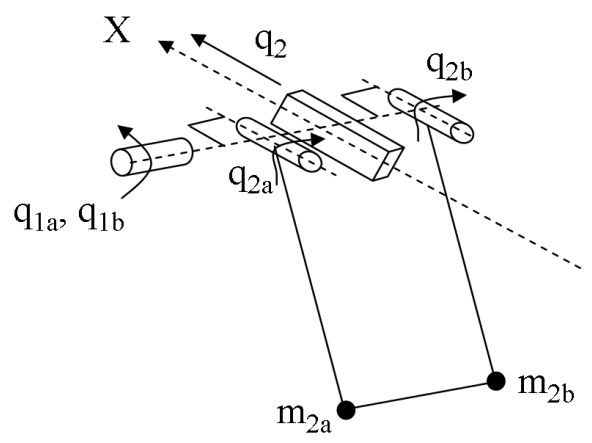

Figure 10: Definitions of the variables at the actuator side for the double link.

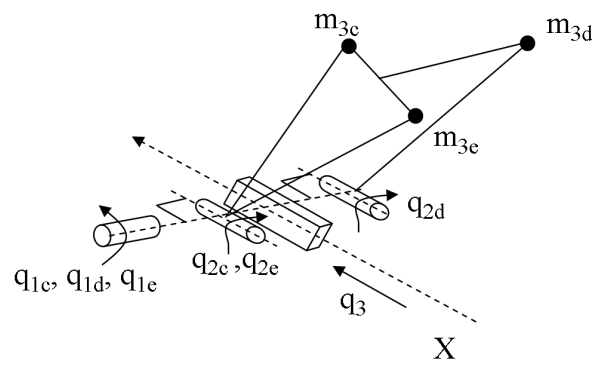

Figure 11: Definitions of the variables at the actuator side for the triple link.

tions $X, Y, Z$. $\left[a_{x} a_{y} a_{z}\right],\left[b_{x} b_{y} b_{z}\right],\left[c_{x} c_{y} c_{z}\right],\left[d_{x} d_{y} d_{z}\right]$, $\left[e_{x} e_{y} e_{z}\right],\left[f_{x} f_{y} f_{z}\right]$ are the coordinates of the points $A, B, C, D, E, F$ in the $\mathbf{T C P}$ coordinate frame.

The $\cos \alpha$ and $\sin \alpha$ equations are given below:

$\cos \alpha=\frac{T_{3 z}-Z}{\sqrt{L_{3 m}^{2}-\left(Y+M_{y}-T_{3 y}\right)^{2}}+\sqrt{M_{x}^{2}+M_{z}^{2}}}$

$\sin \alpha=\sqrt{1-\cos ^{2} \alpha}$

$L_{3 m}$ is the middle length of the triangular-mounted arm 3. $M_{x}^{\prime}, M_{y}^{\prime}, M_{z}^{\prime}$ are coordinates of a vector from a midpoint $M^{\prime}$ between the triangular link coordinates $C$ and $E$ on the platform to the actuator position $\left(T_{3 x} T_{3 y} T_{3 z}\right)$.

$M_{x}^{\prime}=C_{x}+\frac{E_{x}-C_{x}}{2} \quad M_{y}^{\prime}=E_{y} \quad M_{z}^{\prime}=C_{z}+\frac{E_{z}-C_{z}}{2}$

In Figs. 9 - 11 the variables used to define the arm mounting on the carts are shown. The drawing to the left shows the linear actuator variable $q_{1}$ and the two passive joint coordinates $q_{1 f}$ and $q_{2 f}$ for arm 1 . In the middle of the figure, the linear actuator variable $q_{2}$ is defined together with the passive joint coordinates $q_{1 a}$, $q_{1 b}, q_{2 a}$ and $q_{2 b}$ for the parallelogram of arm 2. Because of the parallelogram, the passive angles are related as 
follows: $q_{1 a}=q_{1 b}$ and $q_{2 a}=q_{2 b}$. The drawing to the right shows for arm 3 the linear actuator variable $q_{3}$, the triangular mounted links and the link in parallel with the plane formed by the triangular links. Because of the construction of this arm, the passive joint angles are related (for a nominal model) as follows: $q_{1 c}=$ $q_{1 d}=q_{1 e}$ and $q_{2 c}=q_{2 d}=q_{2 e}$. The subscripts $a$ to $f$ refer to the platform connection points $A$ to $F$, which are defined in Fig. 4.

A prototype of the 3-DOF Gantry-Tau with a triangular-mounted link pair built at the University of Agder, Norway is shown in Fig. 8. The kinematic parameters of the prototype are given below.

$$
\begin{aligned}
& L_{\text {tool }}=0.001 \mathrm{~m} \quad L_{\text {pin }}=0.028 \mathrm{~m} \quad L_{b}=0.03 \mathrm{~m} \\
& R_{p}=0.088 m \quad L_{p}=0.250 m \quad Y_{\text {offs }}=0.125 \mathrm{~m} \\
& L_{1}=1 m \quad L_{2}=1 m \quad L_{3}=1 m \quad Q_{3}=0.42 m \\
& Z_{\text {offs }}=0 \quad Q_{1}=0.5 m \quad Q_{2}=1 m \quad Q_{4}=0 \\
& T_{1 y}=-Q_{1} \quad T_{1 z}=Q_{1} \quad T_{2 y}=0 \\
& T_{2 z}=Q_{2} \quad T_{3 y}=0 \quad T_{3 z}=0 \\
& T_{1 y}^{\prime}=T_{2 y}+Y_{o f f s} \quad T_{1 z}^{\prime}=T_{2 z}-Z_{o f f s} \\
& T_{3 y}^{\prime}=T_{3 y}+Y_{o f f s} \quad T_{3 z}^{\prime}=T_{3 z}+Z_{\text {offs }} \\
& T_{5 y}^{\prime}=T_{3 y} \quad T_{5 z}^{\prime}=T_{3 z}+Z_{o f f s} \\
& T_{2 y}^{\prime}=T_{2 y}-Y_{o f f s} \quad T_{2 z}^{\prime}=T_{2 z}-Z_{\text {offs }} \\
& T_{4 y}^{\prime}=T_{3 y}-Y_{o f f s} \quad T_{4 z}^{\prime}=T_{3 z}+Z_{o f f s} \\
& T_{6 y}^{\prime}=T_{1 y}+Z_{o f f s} \quad T_{6 z}^{\prime}=T_{1 z}-Y_{o f f s}
\end{aligned}
$$

where $Y_{\text {offs }}$ and $Z_{\text {offs }}$ are distances from the base plate to the universal joint in $Y$ - and $Z$-axis, $T_{i y} T_{i z}$ are arm actuator positions and $T_{i y}^{\prime} T_{i z}^{\prime}$ are link actuator positions. $X$-coordinates of the actuator positions $q_{1}, q_{2}, q_{3}$ are defined from general inverse kinematics.

\section{Workspace Evaluation.}

A fully geometric approach to define the maximum workspace of the Gantry-Tau is based on approach presented in Merlet (2000). Fig. 12 shows three circles, one for each arm. The centers of the circles 1, 2 and 3 are located at the points $\left(\left(T_{1 y}+L_{p}+L_{\text {tool }}\right)\left(T_{1 z}+\right.\right.$ $\left.\left.R_{p}+L_{\text {pin }}+\frac{L_{b}}{2}\right)\right),\left(\left(T_{2 y}+Y_{o f f}+L_{\text {tool }}\right)\left(T_{2 z}\right)\right)$ and $\left(\left(T_{3 y}+Y_{\text {off }}+L_{\text {tool }}\right)\left(T_{3 z}\right)\right)$ respectively. The radii of the circles equal to the arm lengths and distance from the connection point on the platform to the $\mathbf{T C P}$ in the $X Z$-plane. The $\mathbf{T C P}$ can only reach points inside of all circles. Fig. 12 also contains three solid lines in the YZ-plane. The TCP is not allowed to move outside of these lines because they indicate the positions of the support framework.

The valid TCP positions are illustrated in a grey colour in Fig. 12. This area $\left(A_{\text {total }}\right)$ can be calculated as a sum of subareas according to:

$$
A_{\text {total }}=A_{1}+A_{2}+A_{3}+A_{4}
$$

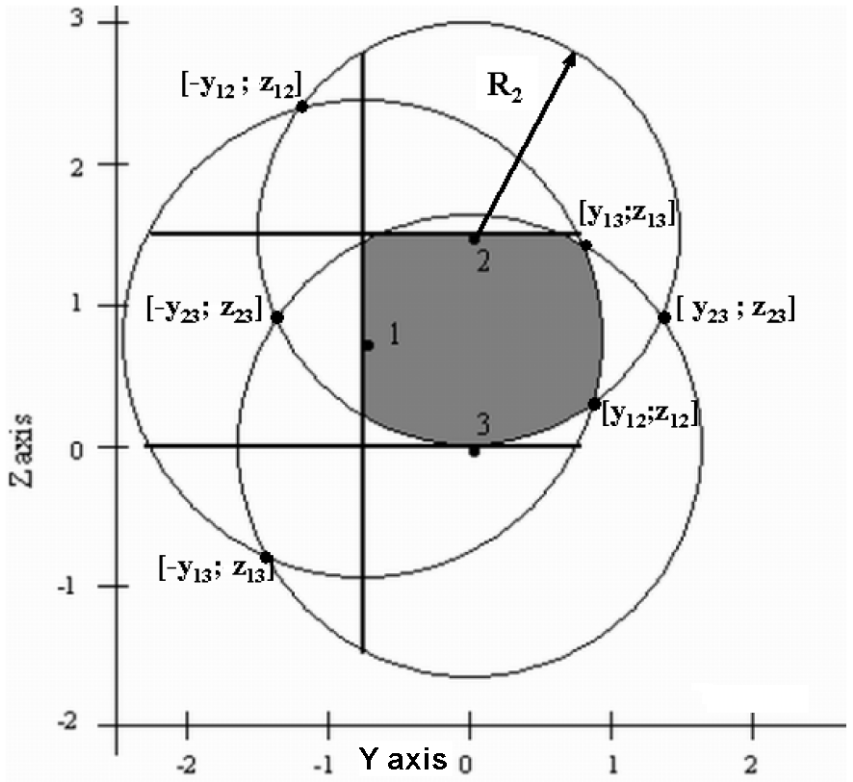

Figure 12: The cross-sectional workspace area of the Gantry-Tau in the YZ-plane.

The subareas are in turn calculated by smaller areas as exemplified for the area $A_{3}$ in Fig. 13. The subarea $A_{3}$ consists of two segments of a circle for small values of $Q_{2}$ because the upper limit of the maximum workspace is partly circular and partly straight line. The first segment of a circle is a segment between points $\left(Q_{1} ; z_{3 Q_{1}}\right)$, $\left(y_{13} ; z_{13}\right)$. The point $\left(y_{13} ; z_{13}\right)$ is a cross-point between circles 1 and 3 . The point $\left(Q_{1} ; z_{3 Q_{1}}\right)$ is a cross-point between the support frame limit $Q_{1}$ and the third arm circle. The second segment of a circle has two limits $\left(-y_{3 Q_{2}} ; Q_{2}\right),\left(y_{3 Q_{2}} ; Q_{2}\right)$ and a centre in the point $\left(\left(T_{3 y}+Y_{o f f}+L_{t o o l}\right)\left(T_{3 z}\right)\right)$. The limits are cross-points between the support frame limit $Q_{2}$ and the third arm circle. At the bigger values of $Q_{2}$ the upper limit is the

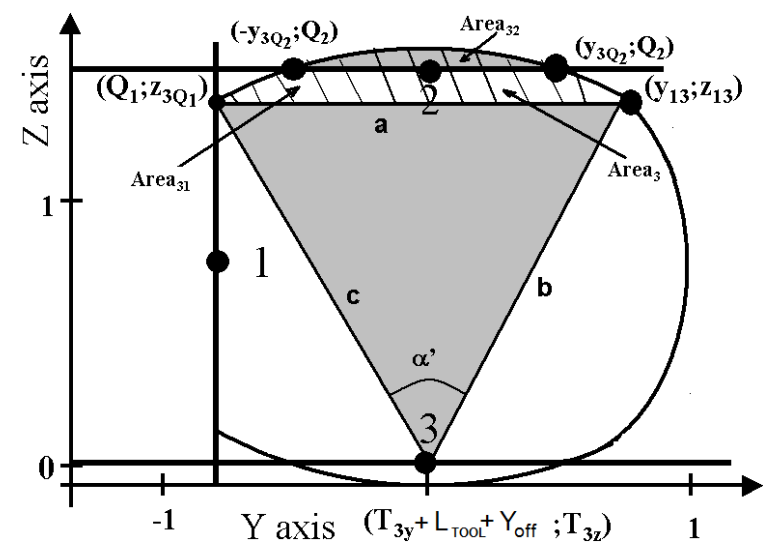

Figure 13: Illustration of workspace Area 3. 


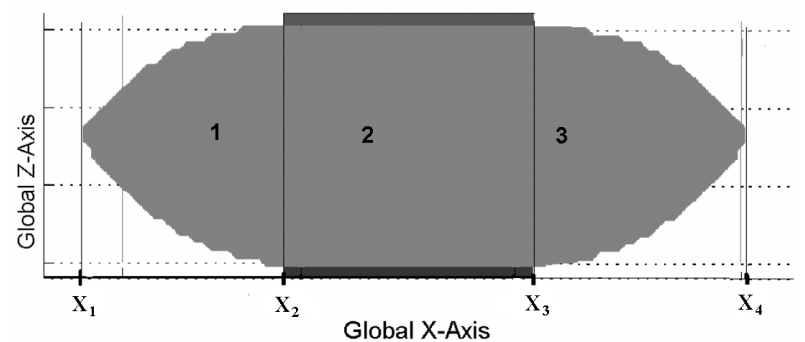

Figure 14: The workspace area of Gantry-Tau machine in the XZ-plane when it is reconfigured to work in both right- and left-handed assembly modes.

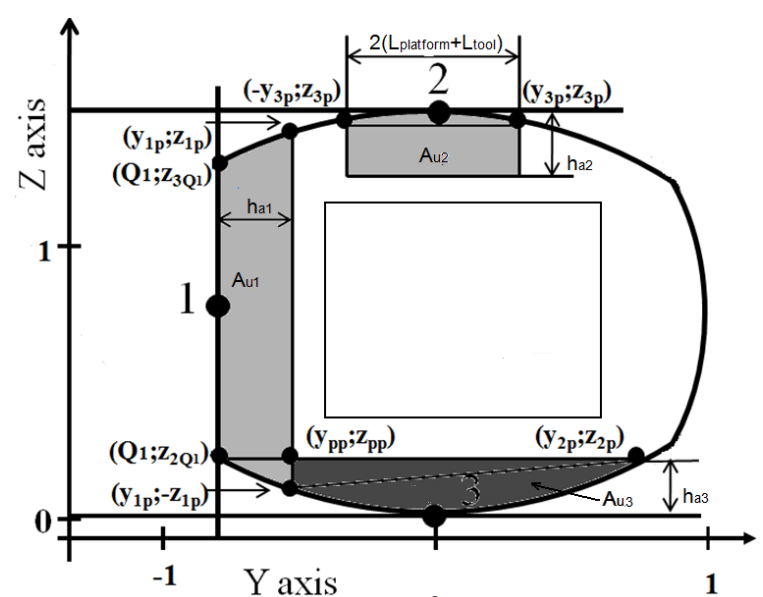

Figure 15: Illustration of the areas where the collisions between the platform and support frame are detected (grey areas). Square in the middle is the user's specified workspace.

circle arc between the points $\left(Q_{1} ; z_{3 Q_{1}}\right)$ and $\left(y_{13} ; z_{13}\right)$. Fig. 14 shows the workspace in the $X Z$-plane, which can be divided into three sections, sections 1 and 3 are outside of the guide ways and section 2 is between the guide ways.

The collisions between the support frame and manipulated platform reduce the maximum workspace. In Fig. 15 the square in the middle defines the user's specified workspace, where all user's requirements are met. The areas where the collisions occur are shown in a grey colour. The lengths of these areas $h_{a 1}, h_{a 2}, h_{a 3}$ and total area $A_{U}$ are expressed as follows.

$$
\begin{aligned}
A_{U} & =A_{U 1}\left(h_{a 1}\right)+A_{U 2}\left(h_{a 2}\right)+A_{U 3}\left(h_{a 3}\right) \\
h_{a 1} & =L_{p}+L_{\text {tool }} \\
h_{a 2} & =R_{p}^{*} \cos \left(\alpha_{\text {min }}\right) \\
h_{a 3} & =\left(\sqrt{L_{3}^{2}-R_{p}^{* 2} \sin ^{2}\left(\frac{2 \pi}{3}\right)}+R_{p}^{*} \cos \left(\frac{2 \pi}{3}\right)\right) \frac{R_{p}^{*} \sin \left(\frac{2 \pi}{3}\right)}{L_{3}} \\
\alpha_{\text {min }} & =\frac{\pi}{2}-\arcsin \left(\frac{Q_{2}}{d e n}\right) i f\left(Q_{2}<\text { den }\right) \\
\alpha_{\text {min }} & =0 \text { if } Q_{2} \geq \operatorname{den} \\
\text { den } & =\sqrt{L_{3}^{2}-R_{p}^{* 2} \sin ^{2}\left(\frac{2 \pi}{3}\right)}+R_{p}^{*} \sin \left(\frac{2 \pi}{3}\right)+R_{p}^{*} \\
R_{p}^{*} & =L_{p i n}+R_{p}+\frac{L_{b}}{2}
\end{aligned}
$$

where $\alpha_{\text {min }}$ is a minimum possible platform orientation angle for the current design. The first area $A_{U 1}$ is located near the actuator $T_{1}$ and found as a summary of three geometric objects. The first object is a segment of a circle between points $\left(Q_{1} ; z_{3 Q_{1}}\right)$ and $\left(y_{1 p} ; z_{1 p}\right)$. The point $\left(y_{1 p} ; z_{1 p}\right)$ is a cross-point between the line $y=Q_{1}-h_{a 1}$ and circle 3 of the maximum workspace. The second segment is found between points $\left(Q_{1} ; z_{2 Q_{1}}\right)$ and $\left(y_{1 p} ;-z_{1 p}\right)$, where the second point is a cross-point between circle 2 and line $y=Q_{1}-h_{a 1}$. The third object (rectangle) is found between points $\left(Q_{1} ; z_{3 Q_{1}}\right),\left(y_{1 p} ; z_{1 p}\right),\left(y_{1 p} ;-z_{1 p}\right)$ and $\left(Q_{1} ; z_{2 Q_{1}}\right)$. Areas $A_{U 2}$ and $A_{U 3}$ are found in a similar way. For more details about the workspace evaluation, link collisions and collisions between the platform and support frame refer to Tyapin (2009).

\section{Static Analysis.}

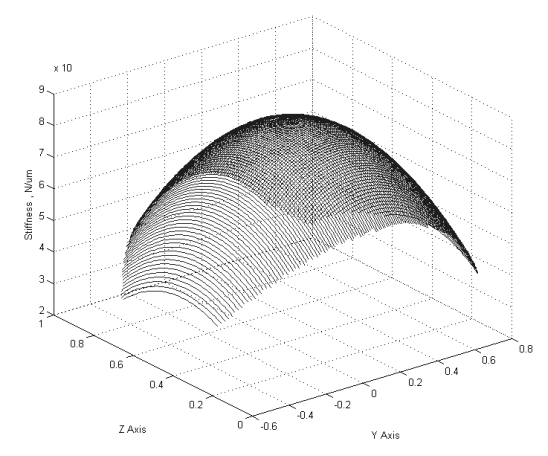

Figure 16: The Cartesian stiffness in the X-direction as function of the $Y$ - and $Z$-coordinates.

Let $\alpha, \beta, \gamma$ be the TCP orientation angles, $l_{i}$ and $F_{i}(i=1, \ldots, 6)$ the six PKM link lengths and link forces. $F_{x}, F_{y}$ and $F_{z}$ are the external Cartesian forces 


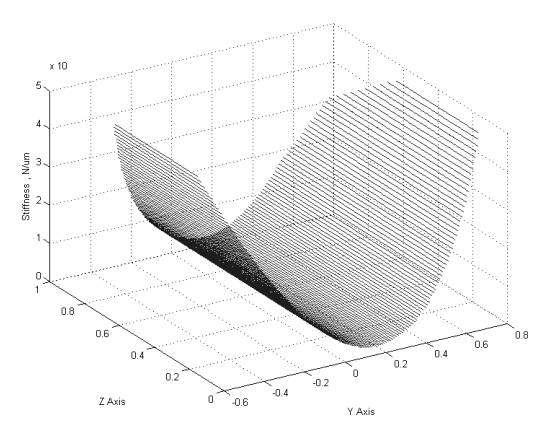

Figure 17: The Cartesian stiffness in the $Y$-direction as function of the $Y$ - and $Z$-coordinates.

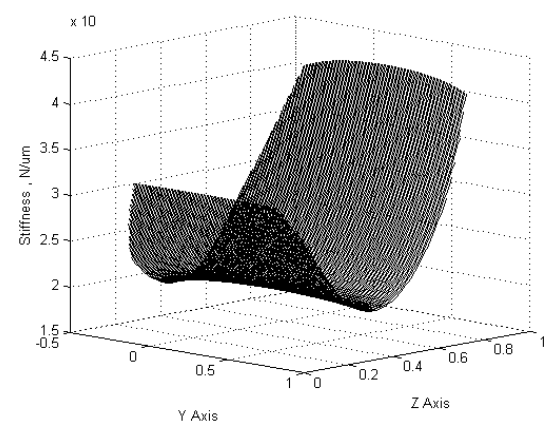

Figure 18: The Cartesian stiffness in the Z-direction as function of the $Y$ - and Z-coordinates.

acting on the TCP. $M_{x}, M_{y}$ and $M_{z}$ are the external Cartesian torques acting on the TCP. The following vectors can then be introduced:

$$
\begin{aligned}
& \mathbf{X}=\left[\begin{array}{lll}
X & Y & Z
\end{array}\right]^{T} \\
& \mathbf{F}=\left[\begin{array}{lll}
F_{x} & F_{y} & F_{z}
\end{array}\right]^{T} \\
& \mathbf{L}=\left[\begin{array}{llllll}
l_{1} & l_{2} & l_{3} & l_{4} & l_{5} & l_{6}
\end{array}\right]^{T} \quad \mathbf{F}_{a}=\left[\begin{array}{llllll}
F_{1} & F_{2} & F_{3} & F_{4} & F_{5} & F_{6}
\end{array}\right]^{T}
\end{aligned}
$$

The relationship between the $\mathbf{T C P}$ forces and the link forces are.

$$
\mathbf{F}=\sum_{i=1}^{6} F_{i} \mathbf{u}_{i} \quad \mathbf{M}=\sum_{i=1}^{6} F_{i} \mathbf{A}_{i} \times \mathbf{u}_{i}
$$

where $\mathbf{u}_{\mathbf{i}}$ is a unit vector in the direction of link $i$ and $\mathbf{A}_{\mathbf{i}}$ is a vector pointing from the TCP to the end-point of link $i$ on the platform. The two equations above can be rewritten using the $6 \times 6$ statics matrix $\mathbf{H}$.

$$
\left[\begin{array}{c}
\mathbf{F} \\
\mathbf{M}
\end{array}\right]=\mathbf{H F}_{a} \quad\left[\begin{array}{c}
\Delta \mathbf{X} \\
\Delta \theta
\end{array}\right]=\mathbf{J} \boldsymbol{\Delta} \mathbf{L}
$$

The Jacobian matrix of the PKM relates changes in Cartesian position $\Delta \mathbf{X}$ and orientation $\Delta \theta$ with changes in the link lengths $\boldsymbol{\Delta} \mathbf{L}$ as shown in Eq. (16)

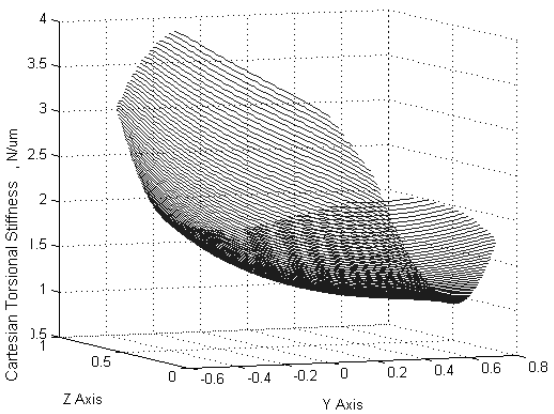

Figure 19: The Cartesian torsional stiffness in the $X$ direction as function of the $Y$ - and $Z$ coordinates.

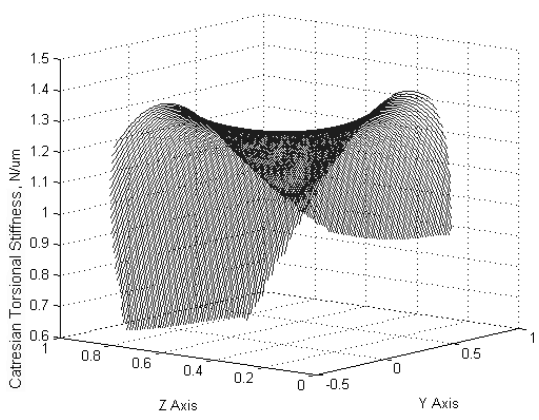

Figure 20: The Cartesian torsional stiffness in the $Y$ direction as function of the $Y$ - and $Z$ coordinates.

(right). In (Gosselin, 1990) the duality between the statics and the link Jacobian for PKMs is presented, ie.

$$
\mathbf{H}^{-1}=\mathbf{J}^{T}
$$

Based on the duality result, the Cartesian stiffness matrix $\boldsymbol{K}$ can be derived as a function of the statics matrix as follows.

$$
\begin{aligned}
{\left[\begin{array}{c}
\mathbf{F} \\
\mathbf{M}
\end{array}\right] } & =\boldsymbol{K}\left[\begin{array}{c}
\boldsymbol{\Delta} \mathbf{X} \\
\boldsymbol{\Delta} \theta
\end{array}\right]=\mathbf{H F} \mathbf{F}_{a}=\mathbf{H} \boldsymbol{K}_{L} \mathbf{\Delta} \mathbf{L} \\
& =\mathbf{H} \boldsymbol{K}_{L} \mathbf{J}^{-1}\left[\begin{array}{c}
\boldsymbol{\Delta} \mathbf{X} \\
\boldsymbol{\Delta} \theta
\end{array}\right]=\mathbf{H} \boldsymbol{K}_{L} \mathbf{H}^{T}\left[\begin{array}{c}
\boldsymbol{\Delta} \mathbf{X} \\
\boldsymbol{\Delta} \theta
\end{array}\right] \\
\Rightarrow \quad & \mathbf{K}=\mathbf{H} \boldsymbol{K}_{L} \mathbf{H}^{T}
\end{aligned}
$$

where $\boldsymbol{K}_{L}$ is a $6 \times 6$ diagonal matrix with the individual link stiffnesses along the diagonal. The matrix $\boldsymbol{K}_{L}$ is presented in (Hovland et al., 2008). The result in Eq. (17) has the benefit that no matrix inversions are required to calculate the Cartesian stiffness at $X-, Y$ and $Z$-coordinates, including coordinates where $\mathbf{H}$ is singular. 


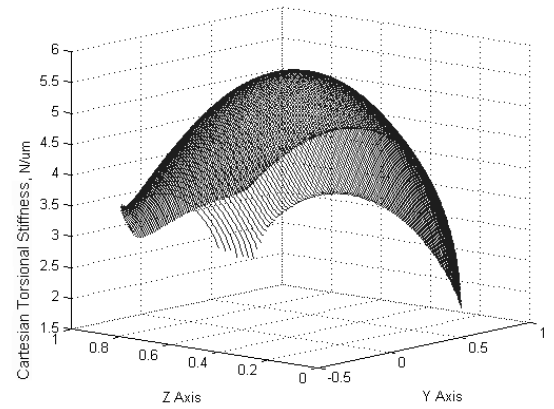

Figure 21: The Cartesian torsional stiffness in the Zdirection as function of the $Y$ - and $Z$ coordinates.

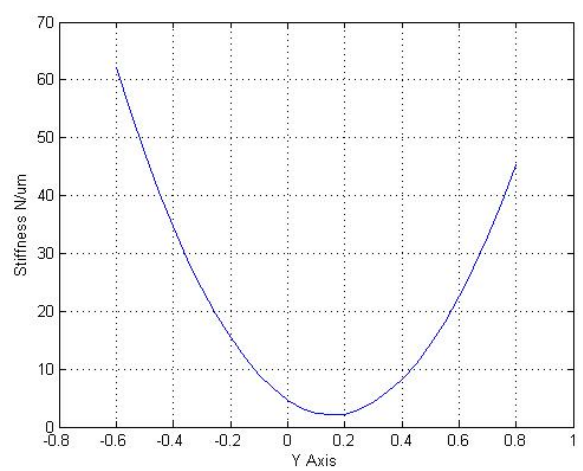

Figure 22: Cartesian stiffness in the $Y$-direction as function of the $Y$-coordinate at fixed $X=1.0$ and $Z=0.4$.

The elements of the matrix $\mathbf{H}$ are the $X$-, $Y$ - and $Z$ components of the vectors pointing from the actuator positions to the points $A, B, C, D, E, F$ on the platform and $X$-, $Y$ - and $Z$ - components of the cross-products of these vectors and vectors pointed from the TCP to the points $A, B, C, D, E, F$ on the platform, see Fig. 4 . The $6 \times 6$ static matrix $\mathbf{H}$ is given below.

$$
\mathbf{H}=\left(\begin{array}{ccc}
A_{x} & \ldots & F_{x} \\
A_{y} & \ldots & F_{y} \\
A_{z} & \ldots & F_{z} \\
(\mathbf{A} \times \mathbf{a})_{x} & \ldots & (\mathbf{F} \times \mathbf{f})_{x} \\
(\mathbf{A} \times \mathbf{a})_{y} & \ldots & (\mathbf{F} \times \mathbf{f})_{y} \\
(\mathbf{A} \times \mathbf{a})_{z} & \ldots & (\mathbf{F} \times \mathbf{f})_{z}
\end{array}\right)
$$

The $Y Z$ functional dependency is applied to find the elements of the static matrix $\mathbf{H}$. Note, that the squares of the elements are required for the Cartesian stiffness.

Stage 1 . In this stage all constants are found. The constants are coordinates of the points $A \ldots F$ on the platform in the TCP coordinate frame, $Y$ - and $Z$ coordinates of the actuators and link lengths. All these

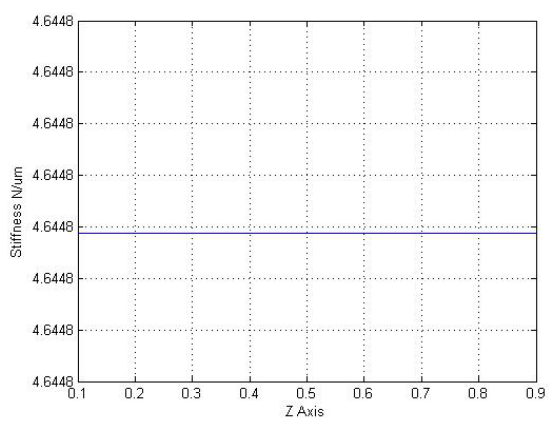

Figure 23: Cartesian stiffness in the $Y$-direction as function of the $Z$-coordinate at fixed $Y=0.0$ and $X=1.0$.

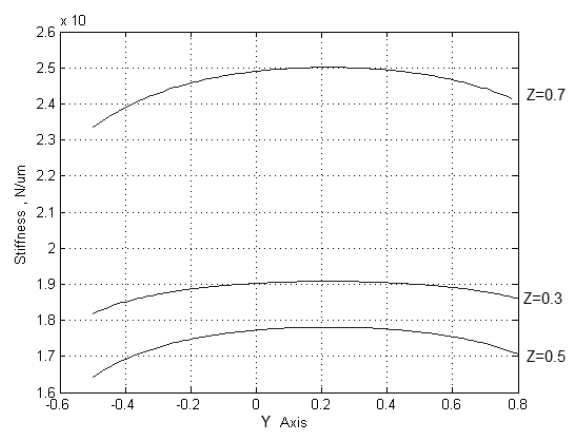

Figure 24: Cartesian stiffness in the Z-direction as function of the $Y$-coordinate at fixed $X=1.0$ and $Z=0.3,0.5,0.7$.

constants are the same for the different $Y Z$-coordinates of the TCP.

Stage 2. In this stage $Y$-coordinate is fixed and $Z$ coordinate is variable. The upper limit of $Z$-coordinate depends on the support frame parameter $Q_{1}$ and the lower limit is 0 . In this paper a calculation algorithm for the first column of the static matrix is presented. The other 5 columns are found in the same way. According to Eqs. (2) and (18),

$$
\begin{array}{r}
H_{11}=\frac{a_{x} \cos \alpha+a_{z} \sin \alpha+X-T_{1 x}^{\prime}}{L_{2}} \\
H_{21}=a_{y}+Y-T_{1 y}^{\prime}=\frac{C_{1 a}^{* *}+Y}{L_{2}} \\
H_{31}=\frac{a_{z} \cos \alpha-a_{x} \sin \alpha+Z-T_{1 z}^{\prime}}{L_{2}}
\end{array}
$$

where $a_{y}, T_{1 y}^{\prime}, a_{z}, a_{x}, T_{1 z}^{\prime}$ are constants, $C_{1 a}^{* *}=a_{y}-T_{1 y}^{\prime}$ is constant. Index $a$ shows the constants related to the point $A$ on the platform. The angle $\alpha$ is expressed as 


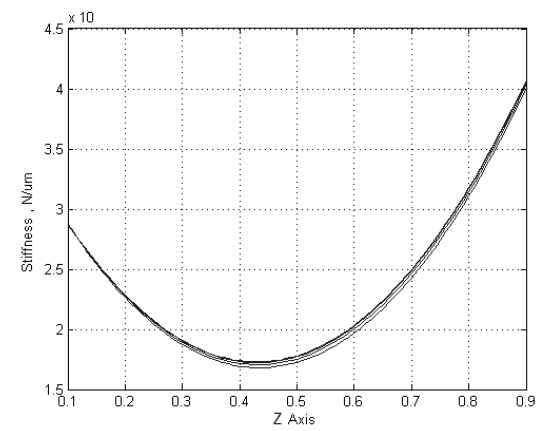

Figure 25: Cartesian stiffness in the Z-direction as function of the $Z$-coordinate at fixed $X=1.0$ and $Y=-0.3,0,0.3,0.6$.

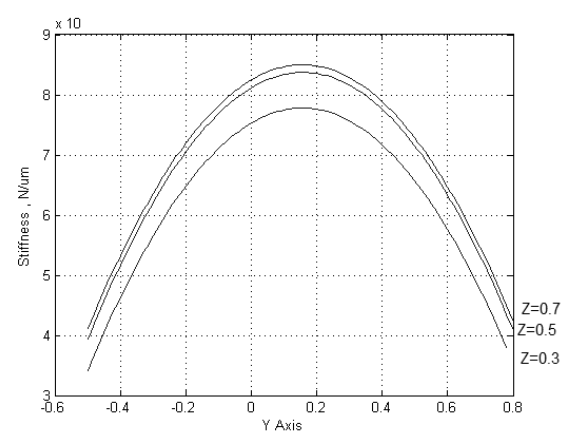

Figure 26: Cartesian stiffness in the $X$-direction as function of the $Y$-coordinate at fixed $X=1.0$ and $Z=0.3,0.5,0.7$.

follows.

$$
\cos \alpha=\frac{T_{3 z}-Z}{\sqrt{C_{1}^{\prime} Y^{2}+C_{2}^{\prime} Y+C_{3}^{\prime}}}
$$

where $C_{1}^{\prime}, C_{2}^{\prime}, C_{3}^{\prime}$ are help constants found from Eq. (3). For the fixed $Y$ Eq. (22) is rewritten as follows.

$$
\cos \alpha=\frac{T_{3 z}-Z}{\sqrt{C_{1}^{\prime \prime}}} \quad \sin \alpha=\sqrt{1-\frac{\left(T_{3 z}-Z\right)^{2}}{C_{1}^{\prime \prime}}}
$$

According to Eqs. (21) and (23), the element $H_{31}$ is expressed as follows.

$$
\begin{aligned}
& H_{31}=\frac{a_{z} \frac{T_{3 z}-Z}{\sqrt{C_{1}^{\prime \prime}}}-a_{x} \sqrt{1-\frac{\left(T_{3 z}-Z\right)^{2}}{C_{1}^{\prime \prime}}}+Z-T_{1 z}^{\prime}}{L_{2}} \Rightarrow \\
& H_{31}=C_{2 a}^{* *}+C_{3 a}^{* *} Z+C_{4 a}^{* *} \sqrt{C_{2}^{\prime \prime} Z^{2}+C_{3}^{\prime \prime} Z+C_{4}^{\prime \prime}}
\end{aligned}
$$

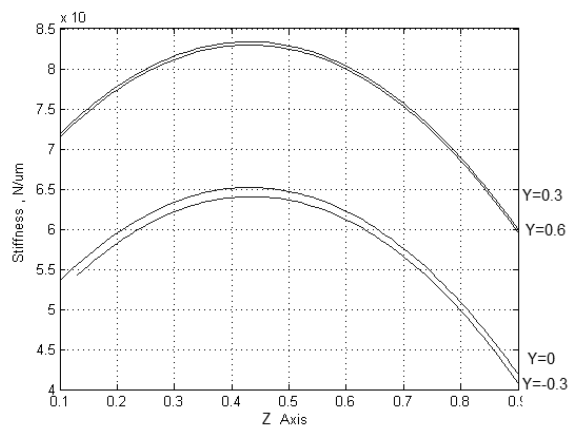

Figure 27: Cartesian stiffness in the $X$-direction as function of the $Z$-coordinate at fixed $X=1.0$ and $Y=-0.3,0,0.3,0.6$.

where

$$
\begin{aligned}
C_{2 a}^{* *} & =\frac{T_{3 z} a_{z}}{\sqrt{C_{1}^{\prime \prime}} L_{2}}-\frac{T_{1 z}^{\prime}}{L_{2}} \quad C_{3 a}^{* *}=-\frac{a_{z}}{\sqrt{C_{1}^{\prime \prime}} L_{2}}+\frac{1}{L_{2}} \\
C_{4 a}^{* *} & =-\frac{a_{x}}{L_{2}}
\end{aligned}
$$

$C_{2}^{\prime \prime}, C_{3}^{\prime \prime}, C_{4}^{\prime \prime}$ are help variables found from Eq. (23).

$$
\begin{array}{r}
X-T_{1 x}^{\prime}=\sqrt{L_{2}^{2}-\left(Y-T_{1 y}^{\prime}\right)^{2}-\left(Z-T_{1 z}^{\prime}\right)^{2}} \\
X-T_{1 x}^{\prime}=\sqrt{C_{4 a}^{\prime \prime} Z^{2}+C_{5 a}^{\prime \prime} Z+C_{6 a}^{\prime \prime}}
\end{array}
$$

According to Eqs. (23) and (19) the element $H_{11}$ of the static matrix $\mathbf{H}$ is given below.

$$
\begin{aligned}
H_{11}= & C_{5 a}^{* *}+C_{6 a}^{* *} Z+C_{7 a}^{* *} \sqrt{C_{2}^{\prime \prime} Z^{2}+C_{3}^{\prime \prime} Z+C_{4}^{\prime \prime}}+ \\
& +\sqrt{C_{4 a}^{\prime \prime} Z^{2}+C_{5 a}^{\prime \prime} Z+C_{6 a}^{\prime \prime}} \\
C_{6 a}^{\prime \prime}= & \frac{L_{2}^{2}-\left(Y-T_{1 y}^{\prime}\right)^{2}-T_{1 z}^{\prime 2}}{L_{2}} \quad C_{5 a}^{\prime \prime}=\frac{2 T_{1 z}^{\prime}}{L_{2}} \\
C_{4 a}^{\prime \prime}= & \frac{1}{L_{2}} \quad C_{5 a}^{* *}=\frac{T_{3 z} a_{x}}{\sqrt{C_{1}^{\prime \prime}} L_{2}} \\
C_{6 a}^{* *}= & \frac{a_{x}}{\sqrt{C_{1}^{\prime \prime}} L_{2}} \quad C_{7 a}^{* *}=\frac{a_{z}}{L_{2}}
\end{aligned}
$$

where a simplification for $X-T_{1 x}^{\prime}$ is given below.

$$
\begin{array}{r}
X-T_{1 x}^{\prime}=\sqrt{L_{2}^{2}-\left(Y-T_{1 y}^{\prime}\right)^{2}-\left(Z-T_{1 z}^{\prime}\right)^{2}} \\
X-T_{1 x}^{\prime}=\sqrt{C_{4 a}^{\prime \prime} Z^{2}+C_{5 a}^{\prime \prime} Z+C_{6 a}^{\prime \prime}}
\end{array}
$$

Stage 3. In this stage $Z$-coordinate is fixed and $Y$ coordinate is variable. The equations for the $\cos \alpha$ is expressed as follows.

$$
\cos \alpha=\frac{C_{1}^{\prime \prime \prime}}{\sqrt{C_{1}^{\prime} Y^{2}+C_{2}^{\prime} Y+C_{3}^{\prime}}}
$$


According to Eqs. (21) and (30) an equation for the element $H_{31}$ is expresses as follows.

$$
\begin{aligned}
H_{31}= & a_{z} \frac{C_{1}^{\prime \prime \prime}}{L_{2} \sqrt{C_{1}^{\prime} Y^{2}+C_{2}^{\prime} Y+C_{3}^{\prime}}}+\frac{Z-T_{1 z}^{\prime}}{L_{2}}- \\
& -\frac{a_{x}}{L_{2}} \sqrt{1-\frac{C_{1}^{\prime \prime 2}}{C_{1}^{\prime} Y^{2}+C_{2}^{\prime} Y+C_{3}^{\prime}}} \\
H_{31}= & \frac{C_{2 a}^{* * *}}{\sqrt{C_{1}^{\prime} Y^{2}+C_{2}^{\prime} Y+C_{3}^{\prime}}}+C_{6 a}^{* * *}- \\
& -\sqrt{\frac{C_{3 a}^{* * *} Y^{2}+C_{4 a}^{* * *} Y+C_{5 a}^{* * *}}{C_{1}^{\prime} Y^{2}+C_{2}^{\prime} Y+C_{3}^{\prime}}}
\end{aligned}
$$

where

$$
\begin{aligned}
& C_{2 a}^{* * *}=\frac{C_{1}^{\prime \prime \prime} a_{z}}{L_{2}} C_{3 a}^{* * *}=\frac{a_{x}^{2} C_{1}^{\prime}}{L_{2}^{2}} \quad C_{4 a}^{* * *}=\frac{a_{x}^{2} C_{2}^{\prime}}{L_{2}^{2}} \\
& C_{5 a}^{* * *}=\frac{a_{x}^{2}\left(C_{3}^{\prime}-C_{1}^{\prime \prime \prime 2}\right)}{L_{2}^{2}} \quad C_{6 a}^{* * *}=\frac{Z-T_{1 z}^{\prime}}{L_{2}^{2}}
\end{aligned}
$$

According to Eqs. (18) and (2)

$$
\begin{array}{r}
H_{11}=\frac{a_{x} \cos \alpha+a_{z} \sin \alpha+X-T_{1 x}^{\prime}}{L_{2}} \\
X-T_{1 x}^{\prime}=\sqrt{L_{2}^{2}-\left(Y-T_{1 y}^{\prime}\right)^{2}-\left(Z-T_{1 z}^{\prime}\right)^{2}} \\
X-T_{1 x}^{\prime}=\sqrt{C_{2 a}^{\prime \prime \prime} Y^{2}+C_{3 a}^{\prime \prime \prime} Y+C_{4 a}^{\prime \prime \prime}}
\end{array}
$$

The element $H_{11}$ of the static matrix $\mathbf{H}$ is given below.

$$
\begin{aligned}
H_{11}= & \frac{C_{7 a}^{* * *}}{\sqrt{C_{1}^{\prime} Y^{2}+C_{2}^{\prime} Y+C_{3}^{\prime}}}- \\
& -\sqrt{\frac{C_{8 a}^{* * *} Y^{2}+C_{9 a}^{* * *} Y+C_{10 a}^{* * *}}{C_{1}^{\prime} Y^{2}+C_{2}^{\prime} Y+C_{3}^{\prime}}}+ \\
& +\sqrt{C_{2 a}^{\prime \prime \prime} Y^{2}+C_{3 a}^{\prime \prime \prime} Y+C_{4 a}^{\prime \prime \prime}} \\
C_{4 a}^{\prime \prime \prime}= & \frac{L_{2}^{2}-\left(Z-T_{1 z}^{\prime}\right)^{2}-T_{1 y}^{\prime 2}}{L_{2}^{2}} \quad C_{3 a}^{\prime \prime \prime}=\frac{2 T_{1 y}^{\prime}}{L_{2}^{2}} \\
C_{2 a}^{\prime \prime \prime}= & \frac{1}{L_{2}^{2}} \quad C_{7 a}^{* * *}=\frac{C_{1}^{\prime \prime \prime} a_{x}}{L_{2}} \quad C_{8 a}^{* * *}=\frac{a_{z}^{2} C_{1}^{\prime}}{L_{2}^{2}} \\
C_{9 a}^{* * *}= & \frac{a_{z}^{2} C_{2}^{\prime}}{L_{2}^{2}} \quad C_{10 a}^{* * *}=\frac{a_{z}^{2} C_{3}^{\prime}}{L_{2}^{2}}
\end{aligned}
$$

Stages 4 . In this stages the elements $H_{41}, H_{51}, H_{61}$ of the static matrix $\mathbf{H}$ are found from the previous stages as a multiplication of two functional dependencies and not presented in this paper because of a limited space and long equations.

The Cartesian stiffness matrix $K$ is a $6 \times 6$ matrix, where the elements $k_{1,1}, k_{2,2}$ and $k_{3,3}$ are the stiffness in the Cartesian $X$-, $Y$ - and $Z$-directions as functions of the $Y$ - and $Z$-coordinates at fixed $X$-coordinate. The diagonal elements of the matrix $K$ are given below.

$$
\begin{array}{r}
k_{1,1}=k_{1} H_{11}^{2}+k_{2} H_{12}^{2}+k_{3} H_{13}^{2}+k_{4} H_{14}^{2}+k_{5} H_{15}^{2}+k_{6} H_{16}^{2} \\
k_{2,2}=k_{1} H_{21}^{2}+k_{2} H_{22}^{2}+k_{3} H_{23}^{2}+k_{4} H_{24}^{2}+k_{5} H_{25}^{2}+k_{6} H_{26}^{2} \\
k_{3,3}=k_{1} H_{31}^{2}+k_{2} H_{32}^{2}+k_{3} H_{33}^{2}+k_{4} H_{34}^{2}+k_{5} H_{35}^{2}+k_{6} H_{36}^{2} \\
k_{4,4}=k_{1} H_{41}^{2}+k_{2} H_{42}^{2}+k_{3} H_{43}^{2}+k_{4} H_{44}^{2}+k_{5} H_{45}^{2}+k_{6} H_{46}^{2} \\
k_{5,5}=k_{1} H_{51}^{2}+k_{2} H_{52}^{2}+k_{3} H_{53}^{2}+k_{4} H_{54}^{2}+k_{5} H_{55}^{2}+k_{6} H_{56}^{2} \\
k_{6,6}=k_{1} H_{61}^{2}+k_{2} H_{62}^{2}+k_{3} H_{63}^{2}+k_{4} H_{64}^{2}+k_{5} H_{65}^{2}+k_{6} H_{66}^{2}
\end{array}
$$

where $k_{i}$ are elements of the matrix $K_{L}$. The Cartesian stiffness in the $X-, Y$-, $Z$-directions are shown in Figs. 16, 17 and 18. The Cartesian torsional stiffness are shown in Figs. 19, 20 and 21.

\subsection{The Cartesian Stiffness in the Y-Direction.}

The weakest stiffness for the Gantry-Tau is the stiffness in the $Y$-direction when the single link is mounted as in Fig. 8. Increasing the stiffness in the $Y$-direction is one of the priorities of the stiffness optimisation for the Gantry-Tau. The Cartesian stiffness in the $Y$-direction as a function of the $Y$-coordinate at fixed $X=1.0$ and $Z=0.4$ is shown in Fig. 22. The stiffness in the $Y$ direction are given in Eq. (38). According to Eq. (20), the general equation for the function $k_{2,2}$ is given below.

$$
\begin{aligned}
k_{2,2}= & C_{1}^{* *} Y^{2}+C_{2}^{* *} Y+C_{3}^{* *} \\
C_{1}^{* *}= & k_{1}+k_{2}+k_{3}+k_{4}+k_{5}+k_{6} \\
C_{2}^{* *} & =k_{1} 2 C_{1 a}^{* *}+k_{2} 2 C_{1 b}^{* *}+k_{3} 2 C_{1 c}^{* *}+k_{4} 2 C_{1 d}^{* *}+ \\
& +k_{5} 2 C_{1 e}^{* *}+k_{6} 2 C_{1 f}^{* *} \\
C_{3}^{* *} & =k_{1} C_{1 a}^{* * 2}+k_{2} C_{1 b}^{* * 2}+k_{3} C_{1 c}^{* * 2}+k_{4} C_{1 d}^{* * 2}+ \\
& +k_{5} C_{1 e}^{* * 2}+k_{6} C_{1 f}^{* * 2}
\end{aligned}
$$

where $C_{1}^{* *}, C_{2}^{* *}, C_{3}^{* *}$ are constants. Eq. (43) is a general parabolic equation.

The approach presented in this Section reduces a computation time effort because the stiffness in the $Y$ direction is found as a parabolic function, where the constants are calculated before the optimisation starts. The Cartesian stiffness in the $Y$-direction as a function of the $Z$-coordinate at fixed $Y=0.0$ and $X=1.0$ is shown in Fig. 23. The stiffness function $k_{2,2}(Z)$ is constant. The stiffness optimisation process requires a maximisation of the minimum stiffness in a given direction. The minimum of the stiffness $k_{2,2}(Y)$ is found 
from Eq. (43) and expressed as follows.

$$
k_{2,2_{\text {min }}}=\frac{4 C_{1}^{* *} C_{3}^{* *}-C_{2}^{* * 2}}{4 C_{1}^{* *}}
$$

\subsection{The Cartesian Stiffness in the Z-Direction.}

The Cartesian stiffness in the $Z$-direction as a function of the $Y$-coordinate at fixed $X=1.0$ and $Z=$ $0.3,0.5,0.7$ is shown in Fig. 24. The Cartesian stiffness in the $Z$-direction as a function of the $Z$-coordinate at fixed $X=1.0$ and $Y=-0.3,0,0.3,0.6$ is shown in Fig. 25.

According to Eqs. (24) and (39), the general equation for the function $k_{3,3}$ is given below.

$$
\begin{aligned}
k_{3,3}= & C_{2}^{* * 2}+C_{3}^{* * 2} Z^{2}+2 C_{2}^{* *} C_{3}^{* *} Z+ \\
& +C_{4}^{* * 2}\left(C_{2}^{\prime \prime} Z^{2}+C_{3}^{\prime \prime} Z+C_{4}^{\prime \prime}\right)+ \\
& +2 C_{2}^{* *} C_{4}^{* *} \sqrt{C_{2}^{\prime \prime} Z^{2}+C_{3}^{\prime \prime} Z+C_{4}^{\prime \prime}}+ \\
& +2 C_{3}^{* *} Z \sqrt{C_{2}^{\prime \prime} Z^{2}+C_{3}^{\prime \prime} Z+C_{4}^{\prime \prime}}
\end{aligned}
$$

where $C_{2}^{* *}-C_{4}^{* *}$ are constants for the given TCP $Y$-coordinate. Due to the additional functions the parabolic functional analysis is not applicable and the Cartesian stiffness function is found as a summation of three functions as shown in Eq. (45). The method presented in Eq. (45) is suitable for the full range of the platform design.

The first derivative of the Eq. (45) is given below.

$$
\begin{aligned}
& \frac{d k_{3,3}(Z)}{d Z}=2 C_{3}^{* * 2} Z+2 C_{2}^{* *} C_{3}^{* *}+C_{4}^{* * 2}\left(2 C_{2}^{\prime \prime} Z+C_{3}^{\prime \prime}\right)+ \\
& +\frac{C_{2}^{* *} C_{4}^{* *}\left(2 C_{2}^{\prime \prime} Z+C_{3}^{\prime \prime}\right)+2 C_{3}^{* *}\left(C_{2}^{\prime \prime} Z^{2}+C_{3}^{\prime \prime} Z+C_{4}^{\prime \prime}\right)}{\sqrt{C_{2}^{\prime \prime} Z^{2}+C_{3}^{\prime \prime} Z+C_{4}^{\prime \prime}}} \\
& +\frac{C_{3}^{* *} Z\left(2 C_{2}^{\prime \prime} Z+C_{3}^{\prime \prime}\right)}{\sqrt{C_{2}^{\prime \prime} Z^{2}+C_{3}^{\prime \prime} Z+C_{4}^{\prime \prime}}}
\end{aligned}
$$

The minimum of the Cartesian stiffness in the $Z$ direction is found from Eq. (46) where the first derivative equals zero or at the boundary of the region. Analysis of these equations shows that the minimum of the stiffness $k_{3,3}$ is found at fixed $X$ and at the limits of the workspace (user's specified area) on the $Y$-axis. $Z$ coordinate is found by solving $\frac{d k_{3,3}(Z)}{d Z}=0$ and both $Z$ - and $Y$-coordinates of the minimum are added to the Eq. (45).

\subsection{The Cartesian Stiffness in the X-Direction.}

The Cartesian stiffness in the $X$-direction as a function of the $Y$-coordinate at fixed $X=1.0$ and $Z=$
0.3, 0.5, 0.7 is shown in Fig. 26. The Cartesian stiffness in the $Z$-direction as a function of the $Z$-coordinate at fixed $X=1.0$ and $Y=-0.3,0,0.3,0.6$ is shown in Fig. 27 and expressed as follows.

$$
\begin{aligned}
k_{1,1}= & k_{1} L_{2}^{2}+k_{2} L_{2}^{2}+k_{3} L_{3}^{2}+k_{4} L_{3}^{2}+k_{5} L_{3}^{2}+ \\
& +k_{6} L_{1}^{2}-k_{2,2}-k_{3,3}
\end{aligned}
$$

The minimum of the Cartesian stiffness in the $X$ direction is found as a minimum of Eq. (47). However, the minimum of the stiffness in the $X$-direction is a function of $Y$ and $Z$. Analysis of Eqs. (43) and (45) shows that the minimum of the stiffness is found at the limits of the user specified workspace in the $Y$ - and $Z$ directions. The minimum of Eq. (47) occurs when $k_{2,2}$ and $k_{3,3}$ are maximal. The functional analysis shows that the Cartesian Stiffness in the $Y$-direction $\left(k_{2,2}\right)$ increases significantly faster than $k_{3,3}$ decreases. In addition, at the $Z$ limits of the user's specified workspace $k_{3,3}$ is maximal. The minimum stiffness in the $X$ direction is found in four cross-points given by $Y$ and $Z$ limits of the user specified workspace.

\subsection{Simulations}

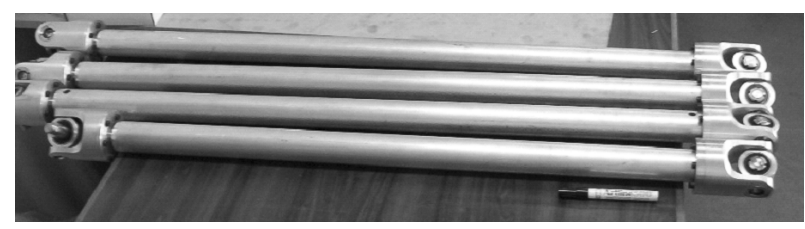

Figure 28: Links and joints of the existing Gantry-Tau prototype.

The minimum, maximum and average Cartesian stiffness in the $X$-, $Y$ - and $Z$-directions of the GantryTau in the entire workspace and the best $70 \%$ of the workspace are given in Table 1 . The physical parameters of the Gantry-Tau are given in Table 2.

The links and joints of the working Gantry-Tau prototype are shown in Fig. 28. Table 3 shows the computational requirements for the four different approaches on the triangular version of the 3-DOF Gantry-Tau PKM. The computing time has been normalised to 1 for the fourth approach presented in this paper.

The method based on the functional dependency is 8250 times faster than the method based on the numerical forward kinematics.

\section{3-DOF Gantry-Tau Design Optimisation}

The Gantry-Tau multi-objective design optimisation problem based on the complex search method is ex- 
Table 1: Cartesian stiffness $(N / \mu m)$ of the 3-DOF Gantry-Tau in the entire workspace and the best $70 \%$ of the workspace.

\begin{tabular}{|c|c|c|c|}
\hline Entire workspace & $\mathrm{X}$ & $\mathrm{Y}$ & $\mathrm{Z}$ \\
\hline Minimum & 26.59 & 2.45 & 19.02 \\
Maximum & 82.82 & 50.90 & 42.29 \\
Average & 65.49 & 13.04 & 26.32 \\
\hline Best 70 percent workspace & $\mathrm{X}$ & $\mathrm{Y}$ & $\mathrm{Z}$ \\
\hline Minimum & 60.56 & 2.45 & 21.45 \\
Maximum & 82.82 & 50.90 & 42.29 \\
Average & 72.76 & 17.48 & 28.89 \\
\hline
\end{tabular}

Table 2: The Gantry-Tau physical parameters.

\begin{tabular}{|c|c|}
\hline Joint weight & $1.0 \mathrm{~kg}$ \\
\hline Joint stiffness & $50 \frac{\mathrm{N}}{\mu \mathrm{m}}$ \\
\hline Link weight & $1.0 \mathrm{~kg}$ \\
\hline Link stiffness & $232 \frac{\mathrm{N}}{\mu \mathrm{m}}$ \\
\hline Platform weight & $5.0 \mathrm{~kg}$ \\
\hline Young's modulus & $70 * 10^{9} \frac{\mathrm{N}}{\mathrm{m}^{2}}$ \\
\hline
\end{tabular}

pressed as follows.

$$
\min \mathbf{F}(\mathbf{p a r})=\left[f_{\text {stif }}(\mathbf{p a r}) f_{\text {qual }}(\mathbf{p a r}) f_{g}(\mathbf{p a r})\right]
$$

Subject to :

$$
\begin{aligned}
& Q_{4}^{L}(\text { par }) \leq Q_{4} \leq Q_{4}^{U}(\text { par }) \\
& Q_{3}^{L}(\text { par }) \leq Q_{3} \leq Q_{3}^{U}(\text { par }) \\
& L_{3}^{L}(\text { par }) \leq L_{3} \leq L_{3}^{U}(\text { par }) \\
& L_{2}^{L}(\text { par }) \leq L_{2} \leq L_{2}^{U}(\text { par }) \\
& L_{1}^{L}(\text { par }) \leq L_{1} \leq L_{1}^{U}(\text { par }) \\
& Q_{1}^{L}(\text { par }) \leq Q_{1} \leq I S_{d t h} \\
& Q_{2}^{L}(\text { par }) \leq Q_{2} \leq I S_{h t h}
\end{aligned}
$$

where par is a vector of the optimisation parameters. Each optimisation parameter has its upper and lower

Table 3: Static stiffness computation time for four different methods.

\begin{tabular}{|c|c|}
\hline Method & Stiffness, Time \\
\hline Numerical forward kinematics & 8250 \\
\hline Jacobian matrix J & 109.9 \\
\hline Static matrix H & 16.5 \\
\hline Functional dependency & 1 \\
\hline
\end{tabular}

limits. The limits of some parameters depend on others. A detailed limits analysis is presented in Tyapin (2009).

A vector of the optimisation parameters par is given below and described in Section 2.

$$
\text { par }=\left[\begin{array}{lllllll}
Q_{1} & Q_{2} & Q_{3} & Q_{4} & L_{1} & L_{2} & L_{3}
\end{array}\right]
$$

The user's specifications included into design optimisation are the minimum required Cartesian stiffness level $k_{m i n}$, minimum distance between two robot's links $L_{C}^{*}$, maximum installation space in the $X-, Y$ and $Z$-directions $I S_{l t h}, I S_{d t h}$ and $I S_{h t h}$, joint angles $J A$, user's specified workspace in the $Y$-direction $U W_{a}$ and in the $Z$-direction $U W_{b}$. The objectives vector $\mathbf{F}$ in Eq. (48) includes workspace, collisions, installation space, statics performances, user's specifications and expressed as follows.

$$
\begin{aligned}
& f_{\text {stif }}(\mathbf{p a r})=\left\{\begin{array}{c}
\frac{k_{\min }}{k_{2,2_{\min }}(\mathbf{p a r})}, \text { if } k_{\min }>k_{2,2_{\min }}(\mathbf{p a r}) \\
1, \text { if } k_{\min } \leq k_{2,2_{\min }}(\mathbf{p a r})
\end{array}\right\} \\
& f_{\text {qual }}(\mathbf{p a r})=\frac{I S}{A_{R}(\mathbf{p a r})-A_{U}(\mathbf{p a r})-\sum_{i=1}^{N U M} \frac{\delta_{i}^{2}}{0.7} A_{C}(\mathbf{p a r})} \\
& f_{g}(\mathbf{p a r})=\sum_{i=1}^{r} G_{i}(\mathbf{p a r}) \\
& A_{C}(\mathbf{p a r})=\left\{\begin{array}{l}
1, \text { if link collisions detected } \\
0, \quad \text { if no link collisions }
\end{array}\right\}
\end{aligned}
$$

where $k_{2,2_{\min }}(\mathbf{p a r})$ is the Cartesian stiffness level presented in Section 4.1, $A_{R}$ (par) is the maximum workspace presented in Section $3, A_{U}$ (par) is the unreachable area caused by the collisions between the platform and support frame presented in Section 3, $A_{C}$ (par) is the link collision parameter and equals 1 if collisions are detected or 0 if there are no collisions for the current workspace cell, 0.7 is a parameter of the sensitivity and equals the workspace and user's specified workspace ratio, $\delta$ is the workspace integration parameter, where the minimum workspace cell equals $\delta^{2}, N U M$ is a number of the workspace cells. $I S$ is the installation space and depends on the parameter $Q_{4}$. For the positive $Q_{4}$ the installation space equals $Q_{2}\left(Q_{1}+Q_{4}\right)$, for negative $Q_{2} Q_{1}$.

Increasing the Cartesian stiffness in the $Y$-direction is the main task of the Gantry-Tau statics optimisation. There are some solutions to increase the stiffness. The first solution is reducing the link lengths while support frame parameters $Q_{1}, Q_{2}$ are fixed. The second solution is increasing the parameters $Q_{1}, Q_{2}$ while the link lengths are fixed. The third solution to increase the stiffness is by shifting the $Y$-position of the actuators 
$T_{2}$ and 3. In this paper the $Y$-position of actuator $T_{2}$ is variable while the actuator $T_{3}$ position is fixed. The fourth solution is a change of the distances between the points $A$ and $B$ or $E, C$ and $D$ on the platform as well as the platform length. The last way to increase the stiffness in the $Y$-direction is to increase $Q_{1}$ while other parameters are fixed. Note, that the support frame parameter $Q_{1}$ defines the $Y$-coordinate of the actuator $T_{1}$. The statics objective in Eq. (49) is a ratio between the required and minimum stiffness for the current design, which indicates the level of the acceptance for the given design.

The quality objective in Eq. (50) includes the workspace, unreachable area, caused by the platform kinematic parameters, installation space and collisions detection. The installation space depends on the support frame parameters. The workspace and unreachable area are the functions of four support frame parameters, individual link lengths and platform kinematics. In Eq. (50) the maximum workspace $A_{R}$ is reduced by the unreachable areas $A_{U}$ on its boundaries and summary of the workspace cells, where the collisions between the links are detected.

The objective function in Eq. (51) keeps the optimisation parameters (constraints) inside of the limits and penalise an infeasible constraints. The constraints handling method is given below.

$$
\begin{aligned}
& g_{i}=0, \quad \text { if } \operatorname{Par}_{i}^{L} \leq \operatorname{Par}_{i}^{c u r} \leq \operatorname{Par}_{i}^{U}(53) \\
& g_{i}=\left(\frac{\operatorname{Par}_{i}^{L}-\operatorname{Par}_{i}^{c u r}}{\operatorname{Par}_{i}^{L}}\right)^{2}, \text { if } \operatorname{Par}_{i}^{\text {cur }}<\operatorname{Par}_{i}^{L}(54) \\
& g_{i}=\left(\frac{\operatorname{Par}_{i}^{\text {cur }}-\operatorname{Par}_{i}^{U}}{\operatorname{Par}_{i}^{U}}\right)^{2}, \text { if } \operatorname{Par}_{i}^{\text {cur }}>\operatorname{Par}_{i}^{U}(55)
\end{aligned}
$$

where $\operatorname{Par}_{i}^{\text {cur }}$ is a current volume of the parameter $i$, $\operatorname{Par}_{i}^{U}$ is the upper limit of the parameter $i$, and $\operatorname{Par}_{i}^{L}$ is the lower limit of the parameter $i$.

The complex search method consists of several stages:

- Generate the initial population of $n$ designs $y_{n}$. As a rule of thumb, the size of the initial population equals $m^{2}$, where $m$ is a number of the optimisation parameters.

- Objectives are evaluated and the worse and the best designs are identified as $y_{j}$ and $y_{k}$ respectively in each iteration.

- The centroid of the remaining design is found as $y_{c}$ in each iteration.

- The worst design $y_{j}$ is mirrored through the centroid $y_{c}$ and a new design is found.

- If the new mirrored through the centroid design continues to be the worst design, it is moved towards the current best design more or less strongly depending on how often this had happened in a row.

In Fig. 29 the implementation of the complex search method is shown, when a new design is found as the worst design mirrored through the centroid. The centroid $y_{c}$ and a new design $y_{j}$ new are defined as follows.

$$
y_{c}=\frac{\sum_{i \neq j}^{n} y_{i}}{n-1} \quad y_{j n e w}=1.3\left(y_{c}-y_{j}\right)+y_{c}
$$

where $n$ is a number of the designs $y$ and $y_{j}$ is the worst design. When a new design is found through the best design, some changes are applied.

$$
\begin{aligned}
y_{c} & =\frac{\sum_{i \neq j}^{n} y_{i}}{n-1} \quad \varepsilon=\frac{n_{0}^{\frac{n_{0}+n_{\text {rep }}-1}{n_{0}}}}{n_{0}+n_{\text {rep }}-1} \\
y_{\text {n new }} & =0.5\left(y_{j}+\varepsilon y_{c}+(1-\varepsilon) y_{k}\right)
\end{aligned}
$$

where $y_{k}$ is the best design, $n_{0}$ is a tuning parameter, normally $4-5, n_{r e p}$ is a number of iterations in a row, where the design $y_{j}$ has been the worse. Parameters $n_{0}$ and $n_{\text {rep }}$ are used to switch the algorithm between the worse and the best designs to find a new one.

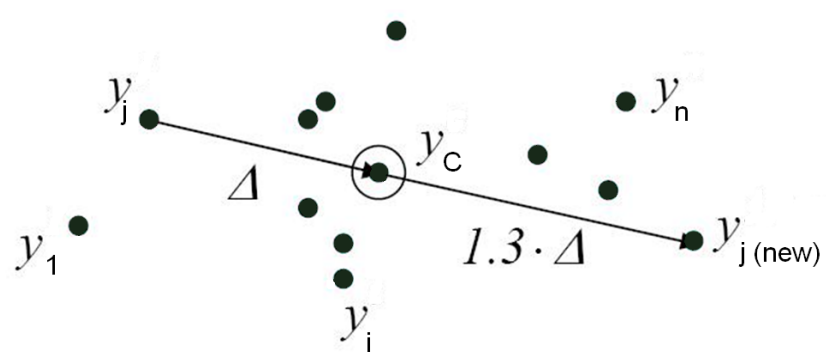

Figure 29: Complex search method. The worst design $y_{j}$ is mirrored through centroid $y_{c}$.

\section{Results}

The final optimisation design parameters of the 3-DOF Gantry-Tau were found using the complex search algorithm and gradient-based function fmincon in integrated Matlab optimisation toolbox. The optimisation results obtained in this paper are summarised in Table 4 and compared with original design parameters, worse and average designs.

The initial population size is 50 randomised designs. The number of evaluations of the objective function was not fixed and optimisation stopped when the difference between the best and worst designs was less than $10^{-3}$. Fig. 30 shows the convergence trend of the main objective function $\mathbf{F}$ as a summary of 3 sub-objectives $\left(f_{\text {qual }}, f_{\text {stif }}\right.$ and $\left.f_{g}\right)$ in 2000 iterations. 


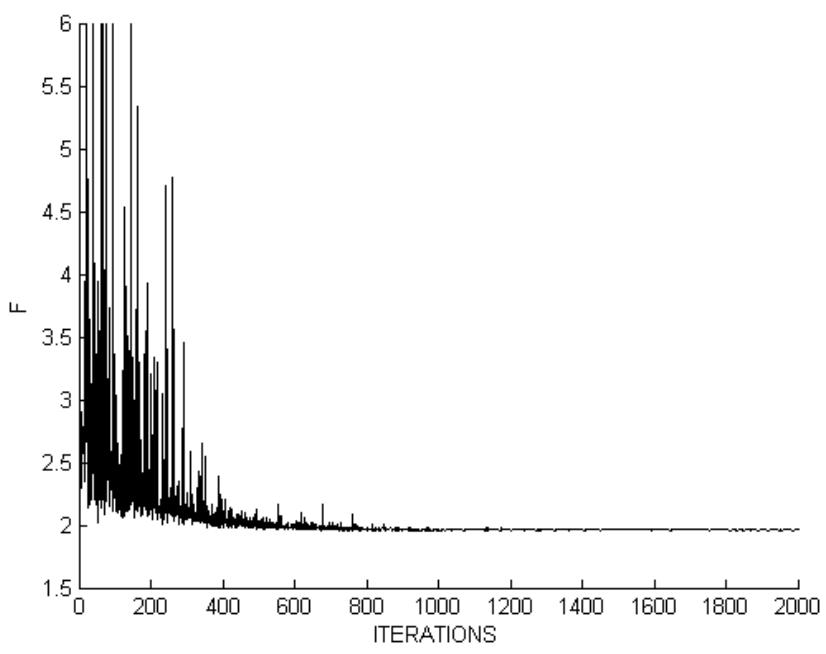

Figure 30: The convergence trend of the main objective function $\mathbf{F}$ as a summary of 3 sub-objectives $\left(f_{\text {qual }}, f_{\text {stif }}\right.$ and $\left.f_{g}\right)$ in 500 iterations.

Table 4: Comparison of the design optimisation results.

\begin{tabular}{|c|c|c|c|c|c|}
\hline Par & Original & $\mathrm{G}-\mathrm{B}$ & Worst & Average & Best \\
\hline$Q_{1}$ & 0.5 & 0.55 & 0.458 & 0.639 & 0.643 \\
\hline$Q_{2}$ & 1 & 1.02 & 1.219 & 0.962 & 0.954 \\
\hline$Q_{3}$ & 0.42 & 0.46 & 0.589 & 0.497 & 0.496 \\
\hline$Q_{4}$ & 0 & 0.168 & 0.087 & 0.215 & 0.229 \\
\hline$L_{1}$ & 1 & 0.94 & 1.151 & 1.184 & 1.108 \\
\hline$L_{2}$ & 1 & 0.95 & 0.953 & 1.038 & 1.038 \\
\hline$L_{3}$ & 1 & 0.94 & 1.252 & 1.02 & 0.976 \\
\hline $\mathbf{F}$ & 3.092 & 2.053 & 4.753 & 2.362 & 1.949 \\
\hline$f_{\text {stif }}$ & 2.451 & 1 & 2.634 & 1.023 & 1 \\
\hline$f_{\text {qual }}$ & 0.641 & 1.053 & 0.710 & 1.162 & 0.949 \\
\hline
\end{tabular}

Figs. 31 and 32 show the sub-objective's $f_{\text {qual }}$ and $f_{\text {stif }}$ convergency trends respectively in 2000 iterations. The sub-objectives of the candidate for the best design are expected as given below. Note that the quality subobjective may vary.

$$
f_{\text {stif }}=1 \quad f_{g}=0
$$

The results would have been difficult to obtain by a manual design, as the the support frame dimension is different from $Q_{1}=2 Q_{2} \approx Q_{3}, Q_{4}=0$ which have been typical manual design choices of the Gantry-Tau in the past. According to Table 4 the the objective function was improved by $36.9 \%$, sub-objectives $f_{\text {qual }}$ and $f_{\text {stif }}$ by $-48 \%$, and $59.1 \%$ respectively. The gradient-based search algorithm can not provide an appropriate results and reach the global optimum in

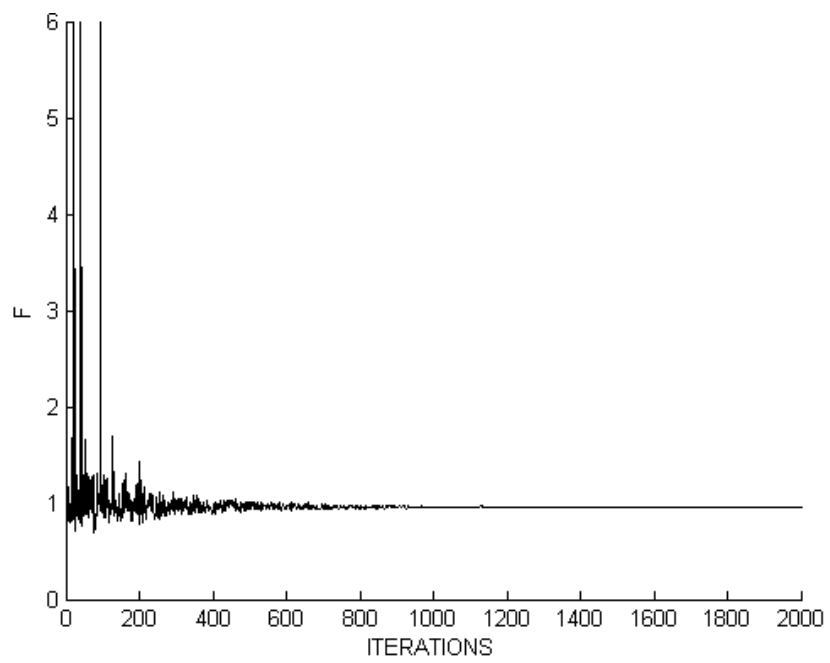

Figure 31: The convergence trend of the sub-objective function $f_{\text {stif }} 500$ iterations.

irregular feasible region. The optimised design was obtained in less than 5 hours on a Pentium Centrino 2 (CPU $2.2 \mathrm{MHz}$ ) computer. Furthermore, the approach proposed in this paper is very simple and easy to be implement for other PKMs.

\section{Conclusions}

One of the main contributions of this paper is the combination of the evolutionary multi-objective methodology based on the complex search algorithm with geometric descriptions of the Gantry-Tau to optimise the parallel kinematic structure. The design optimisation scheme includes the kinematic and elaststatic properties of the PKM. Dimensional synthesis is a part of the design and to obtain the targeted performance at lowest cost, physical design (material selection, bearing design, actuator design etc.) must also be made. The physical design as a part of the Gantry-Tau design optimisation is one of the future research directions.

Multi-objective evolutionary algorithms require a relatively large number of iterations to produce reasonably good approximations of the optimal set of the designs. This has motivated the hybridisation of evolutionary algorithms (global search engines) with local search engines of different types to reduce a computational time effort. An optimisation routine for the platform design according to the required applications would also be a challenging future research topic.

\section{Acknowledgments}

We would like to thank Torgny Brogårdh of ABB Robotics, Sweden for his contributions to this work. 


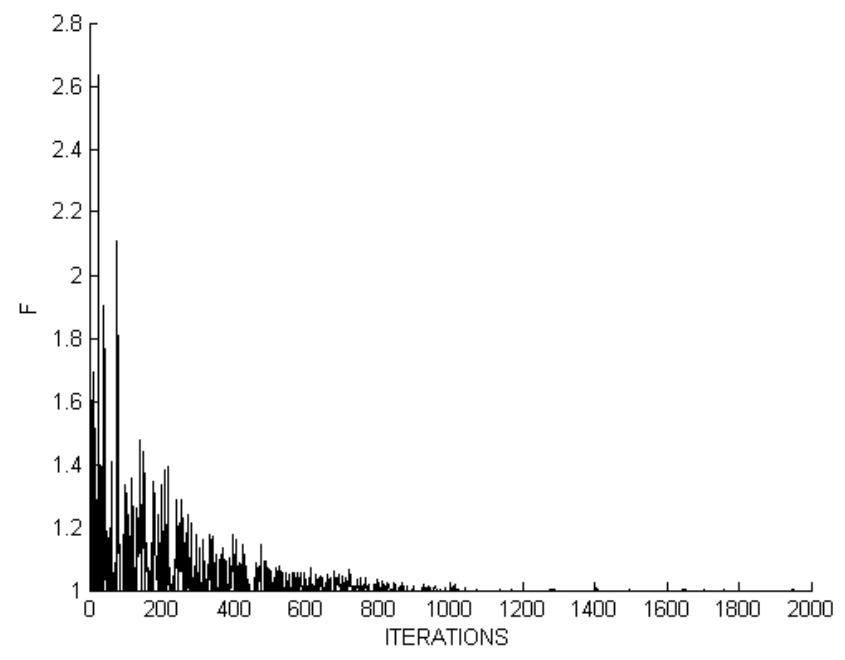

Figure 32: The convergence trend of the sub-objective function $f_{\text {qual }}$ in 500 iterations.

In addition, we want to acknowledge the support from the University of Agder, Norway for providing the resources needed to optimise the Gantry-Tau presented in this paper. The majority of the work presented in this paper was conducted during the first half of 2008 , when I. Tyapin visited the University of Agder.

\section{References}

Bi, Z., Lang, S., Zhang, D., Orban, P., and Verner, M. Integrated design toolbox for tripod-based parallel kinematic machines. Journal of Mechanical Design, 2007. 129:799-807. doi:10.1115/1.2735340.

Brogårdh, T. Design of high performance parallel arm robots for industrial applications. In Proc. of the Symp. Commemorating the Legacy, Works, and Life of Sir Robert Stawell Ball Upon the 100th Anniversary of $A$ Treatise on the Theory on The Screws. University of Cambridge, Trinity College, 2000 .

Brogårdh, T. and Gu, C. Parallel robot development at ABB. In Proc. 1st Intl. Coll. of the Collaborative Research Centre 562. University of Braunschweig, 2002

Brogårdh, T., Hanssen, S., and Hovland, G. Application-oriented development of parallel kinematic manipulators with large workspace. In Proc. 2nd Intl. Coll. of the Collaborative Research Center 562:Robotic Systems for Handling and Assembly. Braunschweig, Germany, 2005 pages 153-170.

Clavel, R. Delta, a fast robot with parallel geome- try. In Intl. Symp. on Industrial Robots. Lausanne, Switzerland, 1988 pages 91-100.

Coello, C. C. Theoretical and numerical constrainthandling techniques used with evolutionary algorithms: A survey of the state of the art. Comp. Meth. in Appl. Mech. and Engnrg, 2002. 191((1112)):1245-1287.

Company, O., Pierrot, F., and Fauroux, J.-C. A method for modeling analytical stiffness of a lower mobility parallel manipulator. In IEEE Intl. Conf. on Robotics and Automat. Barcelona, Spain, 2005 pages $3232-3237$.

Dashy, A., Yeoy, S., Yangz, G., and I.-H.Chery. Workspace analysis and singularity representation of three-legged parallel manipulators. In Proc. 7th Intl. Conf. in Control, Automat., Robotics And Vision. Singapore, 2002 pages 962-967.

Dimentberg, F. The screw Calculus and its Applications in Mechanics. Document FTD-HT-23-1632-67, Foreign Technology Division, Wright-Patterson Air Force Base, Ohio, USA, 1965.

Eberly, D. H. 3D game engine design. Morgan Kaufmann, 2001 page 561.

El-Khasawneh, B. and Ferreira, P. Computation of stiffness and stiffness bounds for parallel link manipulators. In Int. Journal of Machine Tools and Manufacture. Elsevier Science Ltd., 1999 pages 321-342.

Gosselin, C. Determination of the workspace of 6-dof parallel manipulators. ASME J. Mech. Des., 1990. 112:331-336. doi:10.1115/1.2912612.

Gosselin, C. Stiffness mapping for parallel manipulators. IEEE Transactions on Robotics and Automation, 1999. 6(3):377-382. doi:doi:10.1109/70.56657.

Hansen, M. and Andersen, T. A design procedure for actuator control systems using optimization methods. In IEEE The 7th Scandinavian International Conference on Fluid Power. 2001 pages 213-221.

Hansen, M., Andersen, T., and Mouritsen, O. A scheme for handling discrete and continuous design variables in multi criteria design optimization of servo mechanisms. In Mechatronics and Robotics. 2004 pages $234-245$.

Hovland, G., Choux, M., Murray, M., and Brogårdh, T. Benchmark of the 3-dof gantry-tau parallel kinematic machine. In IEEE Intl. Conf. on Robotics and Automat. Roma, Italy, 2007 pages 535-542. 
Hovland, G., Choux, M., Murray, M., Tyapin, I., and Brogårdh, T. The gantry-tau : Summary of latest development at $\mathrm{ABB}$, University of Agder and University of Queensland. In 3rd Intl. Colloquium: Robotic Systems for Handling and Assembly, the Collaborative Research Centre SFB 562. Braunschweig, Germany, 2008 .

Huang, T. and Mei, J. Stiffness estimation of a tripodbased parallel kinematic machine. In International Conference on Robotics and Automation. Seoul, Korea, 2001 pages $3280-3285$.

Li, Y., Chen, S.-F., and Kao, I. Stiffness control and transformation for robotic systems with coordinate and non-coordinate bases. In Intl. Conf. on Roborics and Automat. Washington, USA, 2002 pages 550555 .

Li, Y. and Kao, I. Stiffness control on redundant manipulators: A unique and kinematically consistent solution. In Intl. Conf. on Roborics and Automat. New Orleans, USA, 2004 pages 3956-3961.

Liu, H., Ye, C., Wang, H., and Wei, Y. Stiffness analysis and expirement of a parallel kinematic planer. In Proc. of the IEEE International Conference on Automation and Logistics. Jinan, China, 2007.

Lou, Y., Liu, G., and Li, Z. Randomized optimal design of parallel manipulators. IEEE Transactions on Automation Science and Engineering, 2008. 5(2):223233. doi:doi:10.1109/TASE.2007.909446.

Majou, F., Gosselin, C., Wenger, P., and Chablat, D. Parametric stiffness analysis of the orthoglide. In Intl. Symposium on Robotics. 2004.

Merlet, J.-P. Parallel Robots, page 355. Kluwer Academic Publisher, Solid Mechanics and its Applications, Vol. 74, Dordrecht, Boston, 2000.

Merlet, J.-P. and Daney, D. Leg interference checking of parallel robots over a given workspace trajectory. In Proc. of the IEEE International Conference on Robotics and Automation. Orlando, Florida, 2006.

Pashkevich, A., Wenger, P., and Chablat, D. Design strategies for the geometric synthesis of orthoglide-type mechanisms. Journal of Mechanism and Machine Theory, 2005. 40(8):907-930. doi:10.1016/j.mechmachtheory.2004.12.006.

Pashkevich, A., Wenger, P., and Chablat, D. Kinematic and stiffness analysis of the Orthoglide, a PKM with simple, regular workspace and homogeneous performances. In IEEE Intl. Conf. on Robotics and Automat. Roma, Italy, 2007 pages 549-555.
Pierrot, F., Uchiyama, M., Dauchez, P., and Fournier, A. A new design of a 6 -dof parallel robot. In Proc. 23rd Intl. Symp. on Industrial Robots. 1992 pages 771-776.

Stamper, R., Tsai, L., and Walsh, G. Optimization of a three dof translational platform for well-conditioned workspace. In Proc. of the Int. Conf. Robotics and Automation. New Mexico, 1997 pages 3250-3255.

Teller, S. Distance between two line segments in 3D. Geometric tools, http://www.geometrictools.com, 1998-2008, 2008 .

Tyapin, I. Multi-objective design optimiation of a class of parallel kinematic machines. In Ph.D Thesis. Brisbane, Queensland, Australia, 2009 pages 1-266.

Uchiyama, M., Iimura, K., Pierrot, F., Dauchez, P., Unno, K., and Toyama, O. A new design of a very fast 6-dof parallel robot. Journal of Robotics and Mechatronics, 1990. 2(4):308-315.

Whitney, D. E. Optimum step size control for NewtonRaphson solution of nonlinear vector equations. 1969. 14(4):572-574.

Williams, I., Hovland, G., and Brogårdh, T. Kinematic error calibration of the gantry-tau parallel manipulator. In IEEE Intl. Conf. on Robotics and Automat. Orlando, 2006 pages 4199-4204. 